\title{
Psychophysical interactions with entangled photons: Five exploratory experiments
}

\author{
Dean Radin ${ }^{1 \mathrm{a}}$, Peter A. Bancel ${ }^{2}$ \& Arnaud Delorme ${ }^{1,3}$ \\ ${ }^{1}$ Institute of Noetic Sciences, Petaluma, California, USA; dradin@noetic.org \\ ${ }^{2}$ Institut Métapsychique International, Paris, France; pabancel@free.fr \\ ${ }^{3}$ University of California, San Diego, La Jolla, CA, USA; arnodelorme@gmail.com \\ ${ }^{a}$ Corresponding author: 101 San Antonio Rd., Petaluma, CA 94952 USA
}

\section{Abstract}

Objective: Four laboratory studies and an online experiment explored psychophysical (mind-matter) interactions with quantum entangled photons. Method: Entanglement correlation strength measured in real-time was presented via a graph or dynamic images displayed on a computer monitor or web browser. Participants were tasked with mentally influencing that metric. Results: A statistically significant increase in entanglement strength was obtained in experimental conditions in the four lab studies $(\mathrm{p}<0.02)$, with particularly strong results observed in three studies conducted at the Institute of Noetic Sciences ( $p<0.0002)$. Modest results $(\mathrm{p}<0.05)$ were observed in a high-quality subset of entanglement samples in an online experiment. Control experiments using the same equipment and protocols, but without observers present, showed results consistent with chance expectation in both the lab and online studies. Conclusion: These outcomes suggest that the fidelity of entangled states and the nonlocal resource they entail may be mutable in systems that include conscious awareness. This is potentially of interest for quantum information technologies such as quantum computation, encryption, key distribution, and teleportation. The results are also relevant for interpretations of quantum theory, especially if future studies show that entanglement strength can be mentally modulated above the Tsirelson Bound - the mathematical upper limit predicted by quantum theory. Such an outcome would suggest that quantum theory in its present form does not hold when physical systems interact with certain mental states. The results of these exploratory experiments justify continued investigation of entangled photons as targets of mind-matter interaction. 
Keywords: mind-matter interaction, quantum entanglement, neutral monism, consciousness, psi, psychokinesis, quantum information technologies

\section{Introduction}

Over the last century, research has progressively strengthened the empirical evidence for telepathy, clairvoyance, precognition, and other psychic or "psi" phenomena, while expanding the variety of situations and protocols under which they can be observed and studied. Nearly 20 meta-analyses of classes of psi phenomena support the conclusion that effects observed in controlled laboratory conditions are far beyond chance expectation and are not adequately explained by methodological problems or biases attributable to analytical or publication procedures (Cardeña, 2018; Tressoldi \& Storm, 2021). Publication of topical reviews and metaanalytic reports in mainstream journals has broadened awareness of the research results - and provoked lively exchanges - and this has fostered a more informed and nuanced view of psi in some quarters of the scientific community (Mossbridge \& Radin, 2018; Schooler et al., 2018). In our view, as the collective evidence becomes more persuasive, the focus of scientific inquiry will continue to shift from establishing evidence to gathering psi data that will be useful for theorybuilding. Research on these well-documented forms of anomalous cognition and influence hold considerable promise for advancing scientific knowledge precisely because they expose the limits of our current understanding of Nature. However, along with that promise are unique challenges to experimental investigation.

Amassing evidentiary support for psi is challenging because the conditions for eliciting and enhancing these phenomena are not well understood. Research to help elucidate these conditions is complex because it aims to reveal the psychological, physiological, neurological, and environmental correlates of psi performance. Although such "process-oriented" research is laborious, in principle it is no more challenging than any other form of research on unusual states of consciousness (Corneille \& Luke, 2021; Correa et al., 2022). Much can be learned about the conditions for producing psi effects without directly confronting questions about its fundamental mechanisms or its implications for physics, consciousness, and the mind-matter relationship.

However, in contrast to process-oriented research, experiments to advance our understanding about the underlying nature of psi will need to address more central questions. They include: 1) How do psi phenomena fit in with existing physical theory? 2) What are the implications of these phenomena for our conception of mind and consciousness? 3) What does psi imply for the mind-matter relationship? and 4) What inferences should be drawn about the nature of causality and temporality? These questions are challenging to frame experimentally because each domain is an active area of inquiry in its own right, with its attendant uncertainties 
and controversies, for example in quantum physics (Chiribella \& Spekkens, 2015), consciousness studies (Velmans, 2017), the mind-matter relationship (Stubenberg, 2018), and causality (Mendoza-Martínez et al., 2019; Sheehan, 2006; Svozil, 2018).

In addition, the nature of psi uniquely complicates experimentation. These effects appear to violate everyday constraints of space and time, so it is not possible given our current understanding to determine with certainty from where or even when psi effects originate. Timereversed psi effects are well documented (Mossbridge \& Radin, 2018), and many studies have shown that clairvoyance operates over far distances and under conditions where participants are sequestered in high quality isolation chambers with full electromagnetic shielding (Schwartz, 2015). Hence, it is difficult to know with certainty if effects are associated with the designated participants or with someone else (e.g., the experimenter), or if they occur at times outside of the planned experimental sessions (e.g., when the data are observed). In view of these uncertainties, most psi studies take a practical approach and are analyzed under the assumption that effects do occur within the experimental sessions and are produced by the designated participants. ${ }^{1}$ This is a practical position that we adopt in presenting the experiments discussed here.

Theoretical progress will entail a reckoning with physics, which in turn requires data that informs us about the physical nature of psi effects. However, because the locus of psi is often ambiguous, it is difficult to know if such effects should be sought solely within the cognitive processes of participants, within the systems that are targets of intention, or some combination thereof. That is, many laboratory psi protocols are designed to correlate an intentional mental action or state with a target that is ultimately linked to the outcome of a random event, such as the toss of a die or the output of a truly random number generator (RNG). It has been long recognized that such effects can be alternately interpreted as either anomalous cognition or as anomalous influence (Schmidt, 1987). E.g., correctly guessing a symbol on a hidden card might result from a precognitive glimpse of the future when the card is revealed, or as a mind-matter

${ }^{1}$ There is some evidence for psi-like effects that can be attributed to the experimenter rather than the participants (Kennedy, 1976). But there are also experiments that show fairly constant effect sizes independent of the investigators, and also show differences among selected and non-selected participants. The prime example is the highly successful ganzfeld telepathy experiment, which has been reported by some four dozen different principal investigators (Tressoldi \& Storm, 2021). Similar results have also been observed in the extensive database of micro-psychokinetic experiments (Varvoglis \& Bancel, 2015). 
interaction (also called psychokinetic or PK) influence on the random process used to select that card. Even if the ambiguity can be resolved in favor of PK, data will still be useful for theory to the extent that it carries detailed information on processes that connect to fundamentals of physical theory.

Advances in psi research have often been driven by novel experimental paradigms. Examples include the ganzfeld telepathy experiment, physiological measures of presentiment, and micro-PK studies using RNGs (chapters 15, 17 and 20, respectively, in Cardeña, 2015). Following that tradition, in this paper we report on a novel design using entangled photons as a target system, and we highlight how this kind of experiment may help inform the interface between psi and physics. Photonic entanglement presents not only a simple and extensively studied system within physics, but one that is deeply connected to the essence of quantum mechanics, the most successful theory of physics to date. As Erwin Schrödinger once wrote: "I would not call [entanglement] one but rather the characteristic trait of quantum mechanics, the one that enforces its entire departure from classical lines of thought" (Schrödinger, 1935). If the $20^{\text {th }}$ century conclusion from physics was that Nature is quantum, the conclusion from the first decades of the $21^{\text {st }}$ century is that the essence of quantum nature is revealed through properties of entanglement (Brunner et al., 2014). Accordingly, data that inform us on how psi may interact with entangled states can potentially provide new information for theories both of psi and physics.

Another motivation for our proposal is that psi effects and physical entanglement both exhibit unexplained connections between parts of a system that in turn give rise to influences that are called nonlocal. These notions are well-defined within quantum theory and are descriptive for psi effects, and it will be important to determine whether these parallels are merely coincidental, or if they point to a fundamental aspect of the mind-matter relationship. For example, neutral monism is the philosophical proposition that reality consists of an inextricable relationship between mind and matter (Stubenberg, 2018). Proponents of this view propose that the ability to know a thing (mind), and the thing that can be objectively known (matter), are not truly separate and that both mind and matter are fundamental aspects of a holistic reality, akin to two sides of the same coin. Neutral monism thus suggests that mind is intimately related to foundational concepts in physics, including space and time. This is apropos because recent theories about the structure and existence of spacetime are related to the phenomenon of quantum entanglement (Musser, 2018). Thus, if mental states can be shown to modulate measures of entanglement, then that may inform what has traditionally been considered a purely philosophical position. The possibility of leveraging entanglement to inform propositions about the mind-matter relationship is therefore a principal motivation for introducing the psientanglement experimental paradigm. 
These broader motivations notwithstanding, we turn now to our experiment, which is a concrete example of how the paradigm can be used to address a fundamental question of physics and its relation to psi. The experiment measures photonic entanglement correlations and seeks to determine if a psi effort can modulate the strength of entanglement. A positive result would have practical ramifications because technologies based on quantum entanglement, such as quantum cryptography, rely on the constancy of entanglement measures. Psychic interference with entanglement might compromise these technologies, which are currently considered inviolable. But the experiment also lays the ground for a far-reaching test of quantum physics.

It is well-known that entanglement correlations have an upper limit and are strictly bounded by the mathematics of quantum theory (that limit is called the Tsirelson Bound (Cuffaro, 2018)). What this mathematically-determined bound might suggest about the nature of physical reality is not entirely clear, but it is known that "super-quantum" theories with stronger correlations are conceivable. The important question for psi, then, is whether psi modulations of entanglement strength can exceed the Tsirelson Bound (TB). If it can, then that would have profound implications for both psi and physics (this topic is revisited in the Discussion, later). Thus, an immediate goal of the current work is to determine then if a future psi-TB experiment is feasible.

All of these considerations hinge on a resolution of the locus problem: the ability to ascertain if a psi effect truly corresponds to a change in entanglement strength. That is, controls must determine that an effect is neither an artifact of a PK influence on some part of the experimental apparatus (e.g., the detector efficiency, or mirror alignment), nor due to clairvoyant or precognitive selection of an uncontrolled experimental parameter. Each of these artifactual possibilities can be considered a "loophole" that undermines inferences about psi-induced entanglement modulation. A strong motivation for introducing the psi-entanglement paradigm is that it holds a unique potential for closing, or at least for controlling, these PK loopholes. An indication of how this can work is considered later in the Discussion.

This paper presents four laboratory studies and a similar experiment carried out via the Internet. The goal of these preliminary studies was three-fold: (a) demonstrate a suitable protocol for studying psi effects on entangled photons; (b) explore appropriate methods of analyzing the data; and (c) formulate a measure of effect size to assess the feasibility of the psi-TB experiment. 


\section{Method: Laboratory Experiments}

\subsection{Equipment}

These experiments used a commercial optical system that generates entangled polarization states in pairs of photons (quED, qutools.com, Munich, Germany). The entanglement quantity, denoted as $\mathbf{S}$, is an algebraic combination of measurements first proposed by Clauser, Horne, Shimony, and Holt (Clauser et al. (1969), henceforth CHSH). The CHSH Svalue has become the standard measure of entanglement in paired systems with two-valued measurements (2-qubit entangled states), as is the case for entangled photon polarizations. Its formalism, adapted from Bell's inequality condition for local causality, delineates when correlations imply nonlocal behavior.

In the CHSH scenario, a value of $\mathbf{S} \leq 2$ indicates that the correlations between pairs of photons are behaving in a locally causal manner. In this case, the observed correlations could be explained by a common cause acting on the two photons (a so-called local hidden variable explanation). A correlation strength $\mathbf{S}>2$ indicates that the entangled photons exhibit nonlocal correlations, i.e., pairs of photons are exhibiting correlations that are stronger than any form encountered in classical physics (meaning, constrained exclusively to local interactions). That $\mathbf{S}$ can be greater than 2 has been repeatedly verified to very high degrees of confidence, and that establishes nonlocality as an empirical fact of nature (Aspect et al., 1982; Bub, 2019).

It can also be shown that the mathematics of quantum theory imposes an upper bound, i.e., $\mathbf{S} \leq 2 \sqrt{2}$. This is dubbed the Tsirelson Bound, named after mathematician Boris Tsirelson who first calculated this value (Cuffaro, 2018). This value delimits quantum correlations from hypothetical "super-quantum" correlations that, if shown to exist, would conflict with the mathematical predictions of quantum theory as it is currently understood. The photon pairs produced by the quED apparatus in our experiments operated in the regime $2<\mathbf{S} \leq 2 \sqrt{2}$, which assured that the photons, the "matter" side of these mind-matter interaction experiments, were indeed nonlocally correlated.

The quED apparatus generated entangled photons by passing a blue laser beam through a nonlinear crystal (beta barium borate), which in turn produced pairs of red photons by the process of spontaneous down-conversion. The strength of entanglement, $\mathbf{S}$, was determined by measuring correlations between the photon pairs at different photon polarizations. To achieve this, each photon of a pair of photons was directed by a mirror to a separate, stepper-motor controlled, polarizing filter. The stepper-motors were programmed to cycle repeatedly through a sequence of 16 pairs of polarizer angles, pausing one second for data accumulation at each setting. Photons passing through the polarizers were then carried via fiber optics to a photon 
coincidence counter, which recorded the number of detected pairs. The resulting counts for the 16 settings were then combined by software to output a value for $\mathbf{S}$.

At each polarization setting, the quED transmitted the coincidence counts via TCP-IP on a local area network (LAN) to a remote computer for real-time data processing and generation of display feedback. Each sample of $\mathbf{S}$ was thus based on coincidence counts from 16 consecutive pairs of polarization measurements, and the value of $\mathbf{S}$ was updated each time the polarization settings changed. ${ }^{2}$ For these studies, we placed the quED in a room with no one present in that room while experimental or control runs were underway. Figure 1 shows a typical sequence of $\mathbf{S}$ values obtained over some 800 samples.

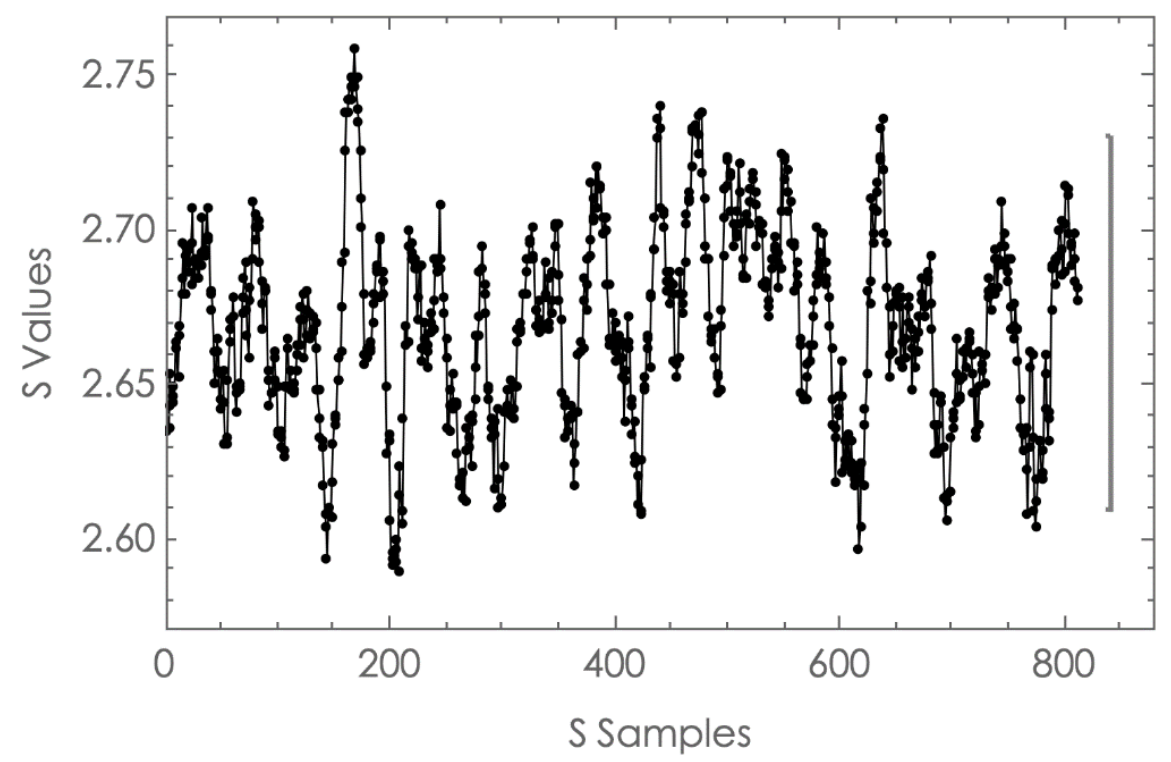

Figure 1. Example of continuously updated $\mathrm{S}$ values returned by the quED system. Note that there are autocorrelations in these samples because each value of $S$ is calculated based on a sequence of 16 coincidence counts. The solid bar on the right side shows the 95\% confidence interval of S based on Poisson counting statistics.

${ }^{2}$ Considered as a time series, the $\mathrm{S}$-values were thus autocorrelated with a dependency that decreased linearly from 1 to zero over the course of 16 lags. 


\subsection{Procedures}

Laboratory experiment 1 (January 2017), experiment 2 (February 2017), and experiment 3 (February 2017) were performed by the first and last authors at the Institute of Noetic Sciences (IONS) in Petaluma, California, USA. Laboratory experiment 4 (August 2017) was an independent collaboration conducted by the second author of the Institut Métapsychique International (IMI) in Paris, France, which used a separate quED system. That study was carried out at the Dechen Chöling retreat center near Limoges, France. All three authors had experienced positive outcomes in previous psi experiments, and as a result they expected that the hypotheses proposed in these studies might be supported. However, because entangled photons had not been used in previous mind-matter interaction studies, their expectations were limited to simply being open to the possibility of observing positive outcomes. Similarly, most of the participants in the laboratory studies were probably open to the same possibility, but no systematic efforts were taken to assess their expectations or prior beliefs.

The general scheme was to compare entanglement $\mathbf{S}$ values under two participant conditions: a "concentrate" condition in which participants were invited to observe a real-time $\mathbf{S}$ value feedback signal and mentally intend for the signal to increase; and a "relax" condition in which feedback was absent and participants were invited to relax and withdraw any intention or attention directed toward the experiment. One possible way to implement this protocol was through a single long period of each attention condition per session and participant. This would have had the advantage of allowing participants an extended time to adapt and settle into each condition. Another possibility was to alternate between brief periods of each condition within a session. The alternation scheme was less settling for participants and made for more complicated analyses. However, it had the advantage of effectively cancelling slow drifts due to variations in laser output or changes in the alignment of the sensitive nonlinear optics. Because slight drifting of the $\mathbf{S}$ value was occasionally observed in test runs, it was deemed prudent to adopt the alternation scheme for all experiments.

\section{Experiments}

In Experiment 1, the controlling computer and the participants were located inside a double steel-walled electromagnetically (EM) shielded chamber (Series 81 Solid Cell chamber, ETS-Lindgren Cedar Park, TX, USA). The quED was in an adjacent room about 5 meters away from the chamber. Because the EM shielded room was electrically sealed, to get the $\mathbf{S}$ samples from the quED into the computer inside the chamber, (a) a translator was used to convert TCP-IP data carried by a CAT- 5 cable (from the quED) into an optical fiber, (b) the fiber was passed through a small data port in the chamber wall, (c) data were converted back into CAT-5, and then (d) the cable was connected to the computer. 
In Experiments 2 and 3, the quED was in the same location but the $\mathbf{S}$ samples were sent via a LAN to a computer in an office in another building about 100 meters away. Experiment 4 did not use an isolation chamber but was otherwise configured similarly to Experiment 1 . The quED was operated at half-power $(25 \mathrm{~mA})$ to conserve the laser in the IONS experiments, and at $35 \mathrm{~mA}$ in the IMI experiment. This resulted in about 1,000 and 2,000 entangled photon pairs per second, respectively. The online experiment is described separately, below.

The IONS configuration used a Windows PC running a custom Matlab (version 2014b, Mathworks, Newton, MA) script that received the $\mathbf{S}$ data and presented participants with instructions and displays to provide graphical and/or audio feedback about the $\mathbf{S}$-values. The IMI experiment used an Apple Mac mini running OSX. The experimental protocols and informed consent forms were approved by the IONS 'Institutional Review Board (IRB number RADD2019_01r072719) and by the IMI's Comité Ethique.

\section{Protocol}

In Experiment 1, participants entered the shielded chamber and were invited to sit in front of the computer that received the $\mathbf{S}$ samples. Next to the computer was an electric candle controlled by an Arduino microcontroller. When the participant was ready to begin, the experimental session was initiated by the experimenter, whereupon a recorded voice played by the computer welcomed the participant. Sessions began with a one-minute rest period, during which $32 \mathbf{S}$ samples were collected without feedback. This took place in near blackout conditions and with no sound. Then, in an alternating fashion, a recorded voice announced the attentional conditions to be adopted by the participant.

First, a voice spoke the phrase get ready, during which $5 \mathbf{S}$ samples were collected without any feedback to the participant. Then the computer announced, now please concentrate, whereupon the next $24 \mathbf{S}$ samples received were presented in graphical form, and by varying the illumination level of an electric candle and the volume of a droning tone. Following that epoch, the recorded voice announced, now please relax, whereupon the electric candle automatically dimmed to a uniform low level, the drone tone was silenced, and 24 more $\mathbf{S}$ samples were silently recorded (and no feedback provided). After 15 repetitions of this get ready-concentraterelax sequence, the session ended. During a session, participants were asked to prepare to focus their attention during the get ready epochs, to focus their attention toward the system while holding the intention to make the candle illumination level and droning volume increase during concentrate epochs, and to withdraw their attention and intention during relax epochs.

Each sample took about 1.5 seconds to collect: 1 second of acquisition time by the photon coincidence counter and 0.5 seconds for the stepper-motors to position the polarization analyzers. Data transmission and computer processing time added about 10 milliseconds for each 
sample. A typical session, consisting of 15 get ready, concentrate, and relax epochs, was thus completed in 20-25 minutes (see Table 1).

In Experiment 2, the same alternating epoch protocol was used, except the feedback for each epoch was randomly selected by the computer to either accurately reflect the $\mathbf{S}$ value or to reverse it. That is, for reversed feedback (during concentrate epochs) the graph, volume of the droning tone, and rise in illumination of the electric candle both varied inversely with the value of $\mathbf{S}$. As in experiment 1, during relax and get ready epochs, no feedback was provided.

The purpose of the reversed feedback condition was to test if the hypothesized effect was due to the act of attention alone, or if the modulation of entanglement strength alternated with the specific direction of mental intention (as mediated through the feedback signal). Participants were blinded to the feedback mode, which ensured a uniform participant experience and avoided confounds (such as unconscious preferences) that may arise when asking people to consciously adopt opposing intentions. A hypothesis that is consistent with the proposal that psi effects correlate with intention states is that the direction of $\mathbf{S}$-value deviations would correspond to the (blinded) feedback mode.

Experiment 3 was similar to Experiment 2, except that all of the $\mathbf{S}$ feedback was uniformly reversed in all sessions, and it involved new participants. Experiment 4 was similar to Experiment 1, with two modifications: 1) the electric candle was replaced by a video animation of a glowing ball in a starry space whose luminosity and size varied with the real-time $\mathbf{S}$ value, and 2) the concentrate period was set to $32 \mathrm{~S}$-value updates instead of 24.

\section{Definitions}

Condition refers to the three attentional instructions assigned to the participant: get ready, concentrate, and relax, as described above.

Trial is the smallest period of data accumulation, corresponding to a single record of data used to generate an $\mathbf{S}$ value. Trial data records provided by the quED included the coincidence photon counts, the orientations of polarization filters, the feedback mode, and other experimental parameters. Recall that the $\mathbf{S}$ value was calculated from the trial's coincidence counts combined with counts from the previous 15 trials.

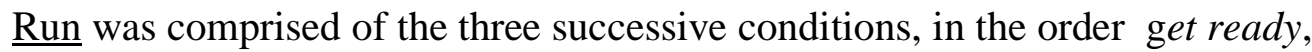
concentrate, and relax. These assignments were announced to the participant by recorded audio at the start of each condition. Each run lasted about one minute and was comprised of either 53 or 61 trials (IONS and IMI, respectively). 
Session was a period of automated data accumulation with a preset number of runs. Sessions typically lasted about 20 minutes and were conducted with a single participant (or for control sessions, no participants).

Table 1. Session parameters for the 4 experiments.

\begin{tabular}{|l|c|c|c|c|}
\hline & IONS_1 & IONS_2 & IONS_3 & IMI \\
\hline Total Sessions & 12 & 10 & 9 & 16 \\
\hline Total Runs & 180 & 154 & 144 & 192 \\
\hline Runs/Session & 15 & $16^{*}$ & 16 & 12 \\
\hline Trials/Session & 827 & $880^{*}$ & 880 & 780 \\
\hline Trials/Condition & $5-24-24$ & $5-24-24$ & $5-24-24$ & $5-32-24$ \\
\hline Feedback mode & Forward & Random per Run & Reverse & Forward \\
\hline Participants & 8 & 1 & 1 & 16 \\
\hline Trial duration & $2 \mathrm{sec}$ & $1.4 \mathrm{sec}$ & $1.4 \mathrm{sec}$ & $1.3 \mathrm{sec}$ \\
\hline Session duration & $27 \mathrm{~min}$ & $20 \mathrm{~min}$ & $20 \mathrm{~min}$ & 16 min \\
\hline Control Sessions & 12 & 10 & 118 & 18 \\
\hline Control Runs & 180 & 160 & 1876 & 364 \\
\hline
\end{tabular}

* One session in Experiment 2 consisted of 10 runs and 540 trials.

\subsection{Hypotheses}

The overall prediction was that $\mathbf{S}$-values associated with concentrate epochs would differ from $\mathbf{S}$-values of relax epochs. The prediction was based on the hypothesis that intentional mental effort can directly influence entanglement, which in turn implies that different efforts would produce different $\mathbf{S}$-values. A natural construction for statistical testing is the numerical difference in $\mathbf{S}$-values for trials of different conditions. In particular, and while recalling that sessions comprise periodic cycles (runs) of get ready-concentrate-relax conditions, we take the difference for trials separated by half a run length. This difference, $\Delta \mathrm{S}$, is calculated for all runs 
in a session or group of sessions. $\Delta \mathrm{S}$ is thus the average difference in entanglement strength between one fiducial point in a canonical run and the paired value half a run cycle away.

Values for $\Delta \mathrm{S}$ are indexed by the fiducial position which we term the lag index. For convenience, the position of the last concentrate trial of the first run is assigned lag $=0$ and $\Delta \mathrm{S}_{l=0}$ is thus the $\mathbf{S}$-value difference of all last trials ${ }^{3}$ of the concentrate and relax epochs, respectively, across all runs and sessions. Calculating $\Delta \mathrm{S}_{\mathrm{l}}$ for successive lags returns a vector, $\boldsymbol{\Delta S}$, and for the analyses presented here, $\Delta \mathbf{S}$ extended to a lag=500 is the basis for statistical tests of a psi entanglement effect. To avoid overcomplicating the description here, further details of this value's construction are provided in the Appendix, but two observations allow for the formulation of hypothesis tests.

First, under the Null hypothesis, each $\Delta \mathrm{S}_{l}$ is a random variable with mean zero and variance determined by the Poisson statistics of the trials' total photon counts. This property is used to devise Monte Carlo simulations to estimate the test statistics' null distributions. Second, by construction, $\mathbf{\Delta S}$ oscillates with a dominant Fourier period equal to the number of trials in a run, which we call L (e.g., see Appendix C and Figure A.1). Therefore, for null data, regression fits of $\Delta \mathbf{S}$ to a Cosine function with period L (i.e., an oscillation) will return fitted amplitudes related to the Poisson variance and phases specific to the dataset.

We wished to formulate hypotheses about deviations in $\mathbf{\Delta S}$ when a psi effect was present, but because the experiment had no precedent it was not clear what type of deviations to test. Too specific a test risks missing the effect, while overly broad tests generally have low power. Our solution was to combine a set of three tests of increasing specificity. For each test statistic, a $p$ value was determined by comparison with its Monte Carlo null distribution. The $p$-values were then Log-summed to create a Fisher chi-squared statistic for which an overall $p$-value was determined by Monte Carlo comparison (described in more detail in Appendix D). The final Fisher $p$-value was then used to test for the effect's significance (with alpha of 0.05).

The three test statistics were as follows: The first took the maximum deviation of $\mathbf{\Delta S}$, $\Delta \mathrm{S}_{\max }=\max \left\{\left|\Delta \mathrm{S}_{l}\right|\right\}$, which was the broadest application of the general hypothesis as stated above. The second test statistic was $\mathbf{A}_{\text {Fit }}$, the fitted Cosine amplitude with period L. This test was

${ }^{3}$ To be precise, at lag=0, $\Delta \mathbf{S}$ is the difference of the last concentrate trials with the 3rd get ready trials (that is, 3 trials after the last trial of the relax epochs). The small offset is due to the brief get ready epochs which add 5 trials to the full run length. 
more specific than the first because its power would increase if an effect was distributed smoothly across runs. The third test was for the Cosine phase. It adds the specificity that effects would closely follow the alternating conditions of concentrate and relax across all runs and participants. In this case it was expected that the phase would lock into a value related to the lag where the effects were strongest. A naïve guess would set the phase locking to lag=0. However, for reasons described in more detail in Appendix B, we used lag=2.5 as the expected value of the Cosine fit phase. As with the other tests, this third test compared the measured phase with its distribution under the Null hypothesis (estimated by Monte Carlo simulation) to return a $p$-value. A fourth hypothesis stated that the psi effect would be of opposite sign when the feedback mode was reversed. These hypotheses are listed in formal terms below (see the Appendix for more details).

Hypothesis 1: $\Delta S_{\max }>0$.

The hypothesis is that $\Delta \mathrm{S}_{\max }$, the maximum unsigned difference in $\mathbf{S}$ values during concentrate vs. relax epochs, would exceed the $95 \%$ confidence interval of its Monte Carlo Null distribution.

Hypothesis 2: $\mathbf{A}_{\mathrm{Expt}}>\mathbf{A}_{\text {Null }}$.

The $\mathbf{\Delta S}$ vector is fit to

$$
\Delta S_{L}=A * \operatorname{Cos}\left(\frac{2 \pi}{L_{R}} L-\phi\right)
$$

where $\mathbf{A}$ is the positive Fourier coefficient, $\phi$ the phase, and $\frac{2 \pi}{L_{R}}$ is the run frequency. The hypothesis states that the fitted parameter $\mathbf{A}$, which models the oscillation of the $\mathbf{S}$ signal that is expected if $\mathbf{S}$ is indeed modulated by intention, will exceed the $95 \%$ CI of its Monte Carlo Null distribution.

Hypothesis 3: Phase locking of $\Delta S$.

This is similar to Hypothesis 2, but more specific in that it predicts that the oscillation is also in phase, with a small lag, with the alternating attentional conditions.

3a) The $95 \% \mathrm{CI}$ of the fit parameter $\phi$ will include the maximum at lag $=2.5$.

3b) The standard deviation of the fit parameter $\phi$ will exceed the $95 \%$ CI of its Monte Carlo Null distribution. The latter is determined by a bootstrap re-sampling of the Monte Carlo data (see Appendix for details). 


\section{Hypothesis 4: $\boldsymbol{\Delta S}$ will correspond to the feedback mode.}

For this hypothesis, analyses of $\mathrm{H} 1, \mathrm{H} 2$ and $\mathrm{H} 3$ were carried out separately on the two types of feedback (forward and reverse). The hypothesis was that the difference between data subsets would be significant at the $5 \%$ level for at least one of the three hypothesis tests.

\subsection{Results}

Across all experimental data, the results of hypothesis tests 1-3 yielded p-values of 0.045 , 0.090 and 0.043 , respectively. Combining these tests (while accounting for test dependencies, as noted below) yielded an overall $\mathrm{p}=0.017$. The feedback hypothesis, $\mathrm{H} 4$, was not supported, and all tests on control data were insignificant. A summary of the test results is shown in Table 2 .

A stark difference was observed between the IONS and IMI datasets, which were acquired separately, under quite different conditions, and with distinct participant pools and environmental settings. The IMI data produced nonsignificant results on all measures, whereas the IONS data were strongly significant on tests $1-3$ and independently provided evidence for an anomalous effect on photon entanglement strength. These tests are described in more detail below, with an emphasis on the significant results of the IONS data. Figure 2 provides a graphical overview of the tests, showing the $\Delta \mathbf{S}$ lag curve, its extremum (per H1), a cosine amplitude fit (H2), and an "S-value Condition Coefficients" curve which models the effect (per H3). See the Appendix A, B, C for details about these measures.

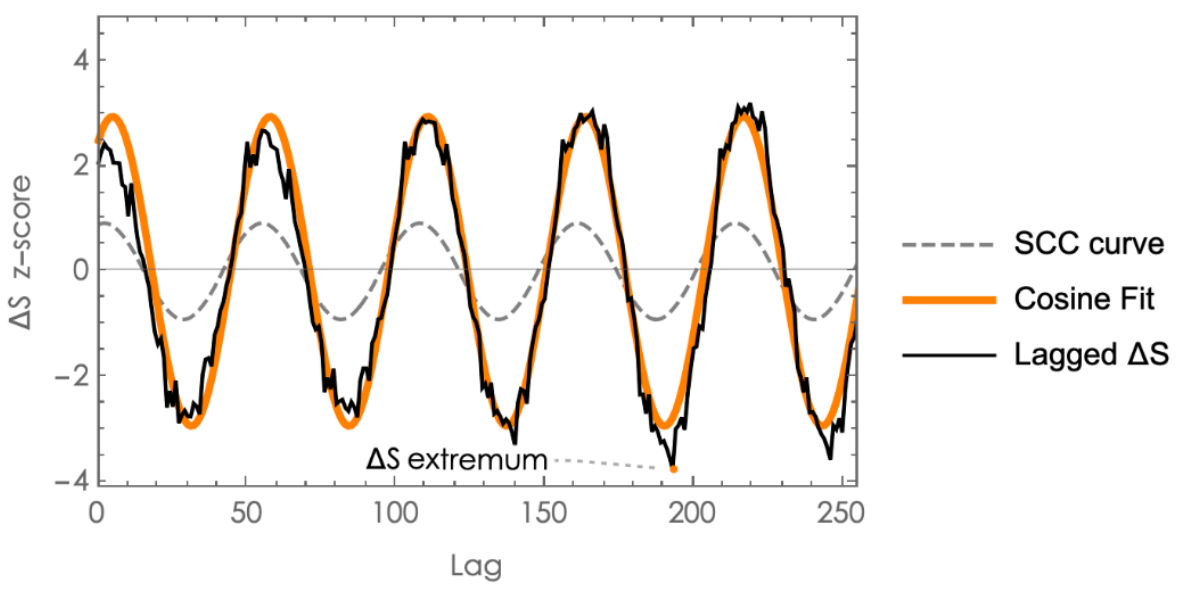

Figure 2. A portion of the $\Delta \mathrm{S}$ lag curve for all IONS data (black trace), as compared to a cosine fit with free parameters of amplitude and phase (orange curve), and a $\mathbf{S}$-value Condition Coefficients (SCC) curve (dotted line). The horizontal axis is the trial lag factor and the vertical axis gives the $\Delta \mathrm{S}$ standard deviation from zero (i.e., the z-score). The $\Delta \mathrm{S}$ extremum of $\mathrm{H} 1$ is also indicated. 
Table 2. One-tailed p-values for tests of H1, H2, H3 and for a composite chi-squared statistic (Fchi), which combines the three values. The p-values were estimated by Monte Carlo simulations performed separately for the IONS and IMI datasets. The independent IONS and IMI $p$-values were combined using a weighted Stouffer Z (inverse normal) procedure to yield global $p$-values for the entire experiment for each hypothesis as well as the combination Fchi. These tests indicate a clear effect in the IONS data and no effect in the IMI data.

\begin{tabular}{|l|l|l|l|l|}
\hline \multirow{2}{*}{ IONS } & \multicolumn{5}{|l|}{ Session $p$-values } \\
\cline { 2 - 5 } & H1 & H2 & H3 & Fchi \\
\hline IMI & 0.014 & 0.003 & 0.008 & $<0.0002$ \\
\hline All Data & 0.54 & 0.54 & 0.41 & 0.58 \\
\hline & 0.09 & 0.045 & 0.043 & $<0.017$ \\
\hline IONS & 0.80 & 0.91 & 0.66 & 0.95 \\
\hline IMI & 0.31 & 0.65 & 0.20 & 0.37 \\
\hline All Data & 0.88 & 0.56 & 0.35 & 0.79 \\
\hline
\end{tabular}

\section{Hypothesis 1: $\Delta S_{\max }>0$.}

The maximum deviation from zero (as a z-score) was extracted (for IONS data this was $\mathrm{z}$ $=-3.77$ at lag 193; see Figure 2). The absolute value of the extremum was then compared to its Null distribution, as determined by Monte Carlo simulation. This yielded a $p$-value estimate of $p$ $=0.014$ for the IONS data. An identical analysis of the IONS control data yielded $p=0.80$.

\section{Hypothesis 2: $\mathbf{A}_{\text {Expt }}>\mathbf{A}_{\text {Null }}$.}

The $\boldsymbol{\Delta} \mathbf{S}$ curve was fit to a cosine function (Figure 2) with free phase parameter and fixed period equivalent to that of the 53-trial experimental run length, $\mathrm{L}_{\mathrm{R}}$. The cosine amplitude was compared to its Null distribution, as determined by Monte Carlo simulation. The $p$-value estimate was $p=0.003$, while control data yielded $p=0.91$. To assure that the optimal fit to $\Delta \mathbf{S}$ did indeed occur at a cosine period of $L_{R}$, the analysis was repeated with cosine periodicities 
ranging from 20 to 90 lags. It was confirmed that the Fourier amplitude was maximized at a cosine periodicity set to the run length, $\mathrm{L}_{\mathrm{R}}$ (Figure 3 ).

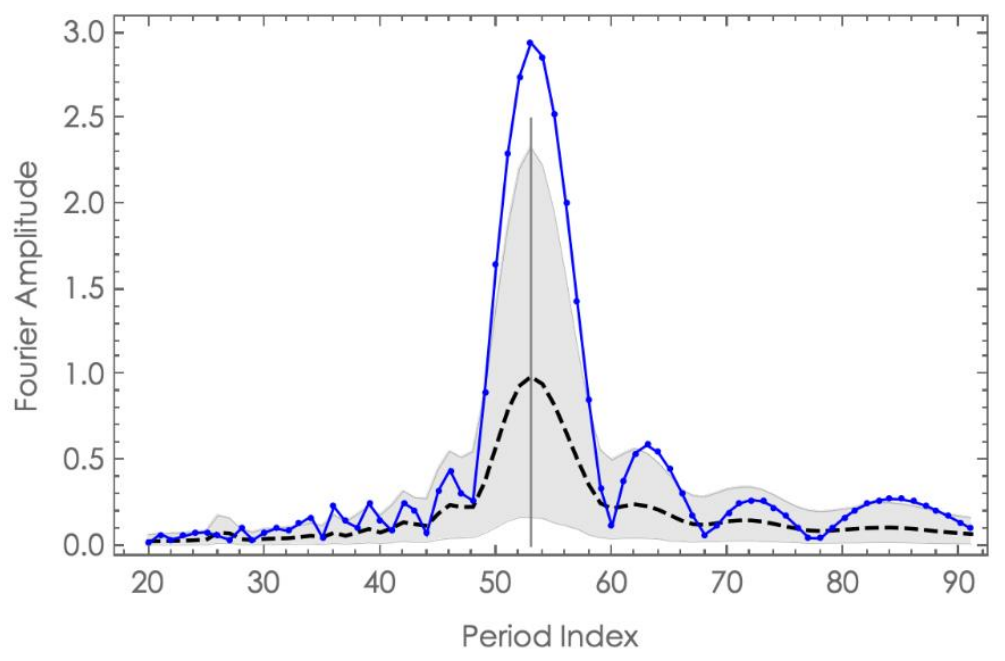

Figure 3. Positive cosine amplitude determined from fits to the $\mathbf{\Delta S}$ lag curve for cosine periodicities ranging from 20 to 90 lags. The peak amplitude occurred at index 53, corresponding to the actual run periodicity. The blue trace is the fitted amplitudes from the IONS data, and the gray envelope is the $95 \%$ Confidence Interval (CI) of the Null distribution as determined by Monte Carlo simulations. The dashed line shows the Null mean values of amplitude fits, indicating that even for no effect, null data will produce a maximum at lag 53 for this analysis. The $95 \%$ CI is relative to the mean, however, and the amplitude fit to the data far exceeds that range.

\section{Hypothesis 3: Phase locking of $\Delta S$.}

Phase locking was tested by first determining that the fitted phase agreed with the model value of lag=2.5, and then testing for a reduced variance of the phase. The reasoning was that an anomalous effect that alternates with the intentional condition ought to lock the fitted phase to the model (see Appendix), thereby reducing the variability of the phase when fit to the data. Alternately, data that happened to yield a false positive result for $\mathrm{H} 1$ and $\mathrm{H} 2$ would not necessarily exhibit phase locking.

The fitted phase and its standard error (in lags) were $4.74 \pm 2.86$. The model maximum, at lag 2.5, was within a standard deviation of the phase parameter. The data and the model were thus aligned to within statistical error. To determine phase-locking, the standard deviation of the fitted phase was compared to its Null distribution obtained from bootstrap resampling of the 
Monte Carlo surrogate data. Further details are in the Appendix. This procedure resulted in $p=$ 0.008 for the phase locking. The same analysis for control data was $p=0.56$.

\section{Combined results across Hypotheses and datasets}

For the IONS and IMI datasets, we combined dependent $p$-values from Hypotheses 1-3 using a modification of Fisher's method. Results from the independent IONS and IMI datasets were combined using a weighted Stouffer Z. The Fisher Chi Square combination of $p$-values, $F c h i$, was given as the sum of the $p$-value logarithms:

$$
\text { Fchi }=-2 \times \sum_{i=1}^{N=3} \log \left[P_{i}\right]
$$

Fisher's method assumes that the quantity Fchi distributes as $\chi^{2}$ with $2 \mathrm{~N}$ degrees of freedom for independent $\mathrm{P}_{\mathrm{i}}$. However, the three hypothesis tests were not independent, thus an empirical distribution for $F c h i$ was determined via Monte Carlo simulation, which incorporated all dependencies into the cumulative distribution function. Using this technique, a combined $p$ value for the IONS data was determined to be $p<0.00021$, and for the control data, $p=0.95$ (Figure 4).

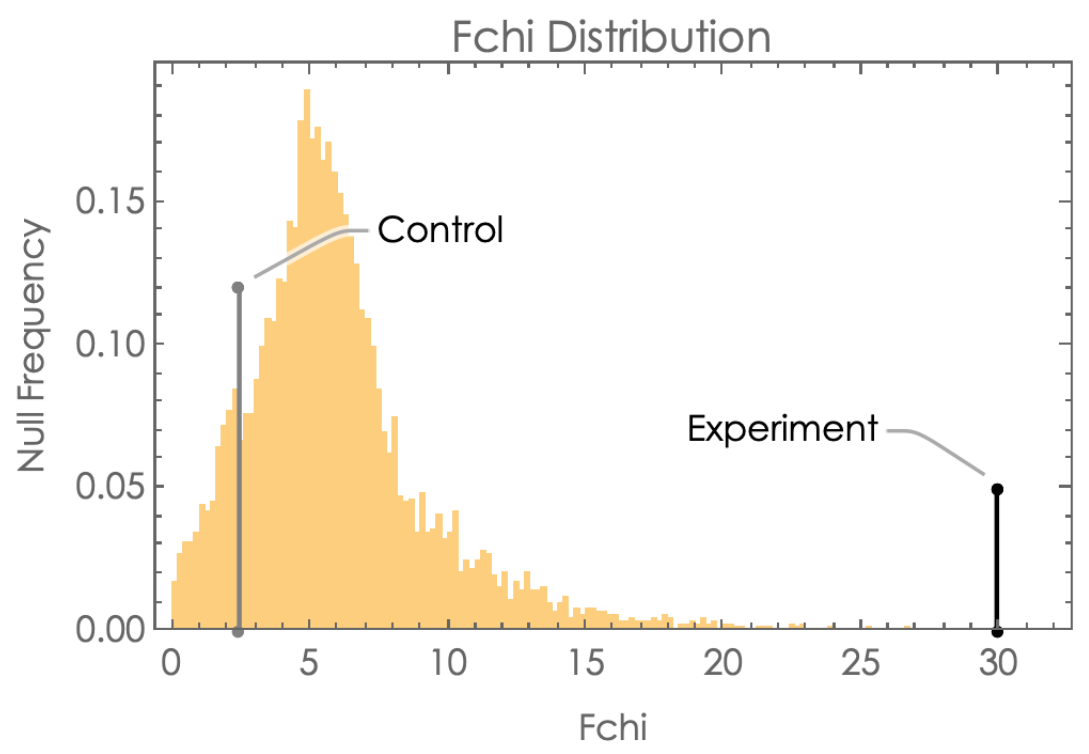

Figure 4. The Null Fchi distribution from combining $p$-values of tests for $\mathrm{H} 1, \mathrm{H} 2$ and H3. The Fchi values for session and control experimental data are indicated. The session $F c h i$ exceeds the largest value of the Monte Carlo distribution, thus the overall $p$-value estimate depended on the number of repetitions of the Monte Carlo and a more accurate $p$-value estimate may well be smaller. 


\section{Hypothesis 4: $\boldsymbol{\Delta S}$ changes sign with feedback mode}

A comparison of feedback modes was performed for the corresponding subsets of the IONS data (IMI data were collected only for the Forward feedback mode). It was found that $\Delta \mathbf{S}$ deviated in the direction of increased entanglement for both feedback modes, and that the deviations were independently significant at the 0.05 level under the combined tests of $\mathrm{H} 1-\mathrm{H} 3$ (see Table 3). Tests of the difference between the two feedback modes were not significant. The hypothesis $\mathrm{H} 4$, that directed intention would correlate with the sign of the effect, was therefore not supported.

Note that the calculation of the $\mathbf{\Delta S}$ lag curve required that the data maintain a regular cycling of polarizer settings throughout each session. Consequently, feedback subsets from experiment 2 were not included in the test of Hypothesis 4 because the feedback mode in that study was randomly assigned from run to run and extracting data by feedback disrupted the continuous ordering of the polarizer settings. The tests of Fchi presented here thus compared data from experiments 1 versus 3 , where the feedback mode was fixed for the duration of each session.

\section{Effect sizes and power estimates}

The data from these experiments allowed us to estimate the experimental effect size as well as to examine the experiment's power under scenarios of different effect sizes. We use a mean-difference type effect size which is defined ${ }^{4}$ as $\mathrm{ES}=\boldsymbol{\Delta} \mathbf{S}_{\mathrm{lag} 2.5} / 4$ and employs the value of $\Delta \mathbf{S}$ at the lag 2.5. An estimate of ES from the experiment was thus obtained by the measured value, $\Delta \mathbf{S}_{\mathrm{lag} 2.5}=0.00575 \pm 0.0024$, whereby $\mathrm{ES}_{\mathrm{Exp}} \approx 0.0014$. With $\mathrm{ES}_{\mathrm{Exp}}$ as input, the Monte Carlo simulations return $\mathrm{ES}_{\mathrm{MC}}=0.00566$, which confirms that a simple Monte Carlo model with ES as input captures the main effect we measured.

${ }^{4}$ The factor of $1 / 4$ is not fundamental and was merely a consequence of how the analyses were constructed; other, equivalent algorithms could have used a different scale factor. 
Table 3. Results of testing Hypothesis 4 among the IONS experiments. Table entries for the values of $\Delta \mathbf{S}$ at lag $\mathrm{L}=2$ and the corresponding standard deviations from zero $\left(\mathbf{Z}_{\Delta \mathrm{S}}\right)$ were calculated from data of all three IONS experiments. The $F c h i$ values and corresponding $p$-values for the IONS Forward and Review modes were calculated from experiments 1 and 3 only.

\begin{tabular}{|l|c|c|c|c|}
\hline \multirow{2}{*}{} & \multicolumn{4}{|c|}{ Session Data } \\
\hline \multirow{2}{*}{ All IONS } & $\Delta \mathbf{S} \cdot 10^{-3}$ & $\mathbf{Z}_{\Delta \mathbf{S}}$ & Fchi & Fchi p-value \\
\cline { 2 - 5 } & $5.7(2.4)$ & 2.43 & 29.9 & $<0.00021$ \\
\hline IONS Forward & $5.0(2.9)$ & 1.71 & 17.0 & 0.01 \\
\hline IONS Reverse & $7.8(5.1)$ & 1.54 & 13.4 & 0.04 \\
\hline Fwd - Reverse & $-3.3(5.8)$ & -0.56 & $6.6(4.7)$ & 0.15 \\
\hline
\end{tabular}

\begin{tabular}{|l|c|c|c|c|}
\hline & \multicolumn{4}{|c|}{ Control Data } \\
\hline \multirow{2}{*}{ All IONS } & $\Delta \mathbf{S} \cdot 10^{-3}$ & $\mathbf{Z}_{\Delta \mathbf{S}}$ & Fchi & Fchi Pval \\
\cline { 2 - 5 } IONS Forward & $1.8(1.4)$ & 1.22 & 2.4 & 0.89 \\
\cline { 2 - 5 } IONS Reverse & $0.1(1.7)$ & 0.04 & 1.1 & 0.97 \\
\hline Fwd - Reverse & $2.7(2.9)$ & 0.95 & $1.5(4.7)$ & 0.69 \\
\hline
\end{tabular}

The Monte Carlo model uses a constant effect, neglecting any inter-session or inter-run variability. Despite this simplification, the model nevertheless allows for useful power estimations of the various tests and analyses we have presented. A summary plot is shown in Figure 5. The estimates show that the Fchi statistics which include $\mathrm{H} 3$ give a substantial power increase over the tests taken individually or in pairs without H3. Although Figure 5 also shows that a simple test of $\mathbf{\Delta S}$ at lag $=2$ has the highest power, the test assumes no variability among sessions and runs, as well as prior knowledge of the optimal lag for testing. None of these 
conditions are likely to hold in a real experiment. Thus, for real data on a new experiment, we expect that the Fchi test that we developed and used for analysis would have the highest power among the approaches outlined.

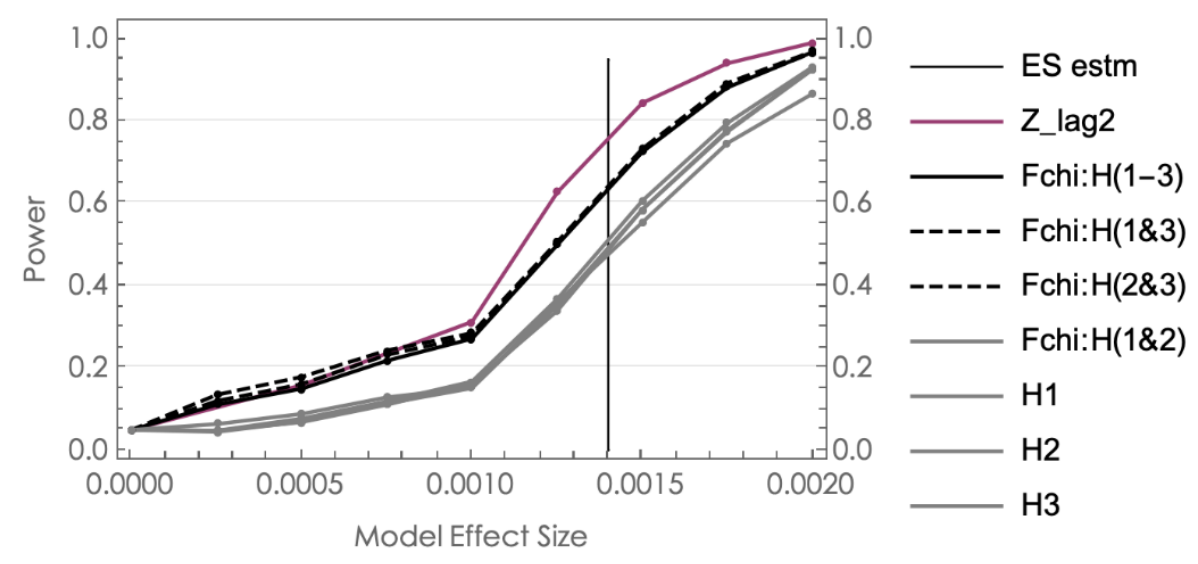

Figure 5. Power estimates for hypothesis tests and combinations of tests, as estimated by constant effect Monte Carlo simulations. The horizontal axis is the model ES, as described in the text, and the vertical axis is the statistical power for significance at an alpha level of 0.05 . The Fchi power curves refer to combinations of hypothesis tests, $\mathrm{H} 1, \mathrm{H} 2$ and $\mathrm{H} 3$. Combined tests that include the phase variance tests of $\mathrm{H} 3$ have higher powers than any of the tests individually, or combined tests without H3. Z_lag2 is the power of the $\Delta \mathbf{S}$ z-score at lag $=2$, for this model. The vertical bar indicates the estimated ES of the experiment (an estimate of the ES uncertainty is $\sim 0.0004$ at onesigma).

\section{Method: Online Experiment}

In this version of the experiment, $\mathbf{S}$ samples were sent over the Internet from the quED device located in the IONS laboratory to an Amazon cloud server (Figure 6). The experiment was programmed using a combination of PHP, Javascript, Ajax, and MySQL, and the web server was a Ubuntu Linux virtual machine hosted by Amazon. The server operated continuously and assigned each incoming $\mathbf{S}$ sample to either a concentrate or relax epoch, where each epoch was uniformly 24 samples in length. Each sample took an average of about 2 seconds to process, approximately $500 \mathrm{msec}$ longer than in the laboratory tests, due to Internet transmission and server processing time. The two types of epochs automatically alternated after collecting 24 samples. 


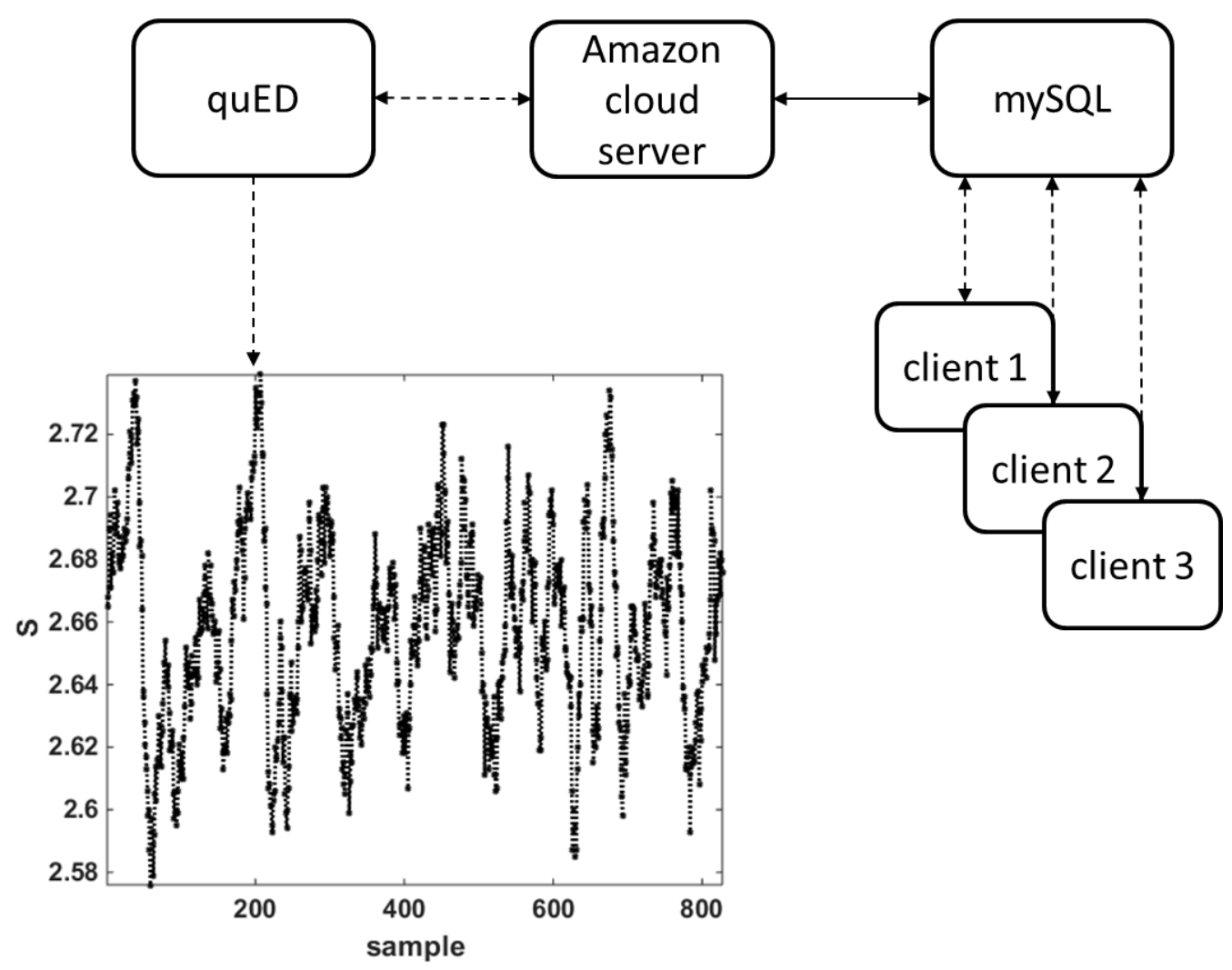

Figure 6. Online experiment infrastructure. All data, including the $\mathbf{S}$ samples and the attention assignments, were stored in a mySQL database. A SurveyMonkey.com questionnaire was used to collect information about the participants. User logins were handled via a Google login process, and user identities were anonymized via md5 encryption.

\subsection{Procedure}

When a user navigated to the website entangledphotons.us, the browser led the participant through a login process to create a unique ID, then it presented options for two types of feedback displays. One was a graph similar to that used in the laboratory experiments; the other was a "bubble cloud" display that looked like a collection of randomly bouncing colored bubbles. The feedback provided in both displays was accurate in the sense that an increase in $\mathbf{S}$ was reflected by a line in the chart going up, or by the randomly moving bubble cloud coalescing into a pleasing toroid shape. Besides the visual feedback, a whistling wind sound was also played, where the pitch of the wind corresponded to the height displayed in the graph or to the degree of bubble coalescence. 
As in the laboratory studies, during concentrate epochs the feedback was displayed, and during relax epochs the feedback display was removed and replaced by a grey rectangle. Unlike in the laboratory studies, there was no get ready condition.

If the web server failed to receive an $\mathbf{S}$ value from the quED within a few seconds, to avoid freezing the feedback display the server automatically switched to a pseudorandom number generator to produce "pseudo-S samples" generated uniformly at random between the values 2.1 and 2.3. These pseudo-samples were noted in the database, but the feedback presented to the participants was not altered to allow the website to continually remain active. When the quED failed, the device was manually reset to continue to generate entangled photons.

Because the data from the quED were automatically sent to a database on the Amazon cloud server, by design this allowed many people to participate in the experiment at the same time. That is, the same feedback display under the same attention condition could be viewed on many web browsers. This provided a way to explore the effects of multiple people attempting to simultaneously influence the entangled photons. When no observers were engaged with the system, the quED data continued to generate $\mathbf{S}$ samples and send them to the database, providing an ongoing no-observation control condition.

When a candidate participant clicked on a button in their browser to start the experiment, that act was taken as a positive consent to participate in the study. The instructions were to mentally attend to the feedback with intention to increase entanglement strength during the concentrate epochs, and to withdraw attention and intention during the relax epochs.

\subsection{Analysis}

The analytical methods used to evaluate the data produced in the online experiment differed from the methods used in the laboratory studies for two reasons. First, by design multiple people could conduct the study at the same time, so besides examining overall results, the data could be examined separately for none (the control condition), one, two, or more simultaneous participants. Second, the quED device was not designed to operate continuously for long periods of time, so the quality of photon entanglement began to degrade over time. The change in $\mathbf{S}$ values and occasional failure of the quED system required several preprocessing steps to transform the recorded data into useable samples.

Figure 7 shows the full set of data recorded in this experiment. Samples in the range $2.1 \leq$ $\mathbf{S} \leq 2.3$ were generated by the pseudorandom algorithm. Each such sample was marked in the database to distinguish it from an $\mathbf{S}$ value. A second type of sample was noise, identified as values below 2.1 or above 2.83 (the Tsirelson Bound). This occurred when $\mathbf{S}$ values were either 
not transferred correctly to the cloud server, or when spurious values were generated by the quED. ${ }^{5}$ A third category was valid $\mathbf{S}$ samples.

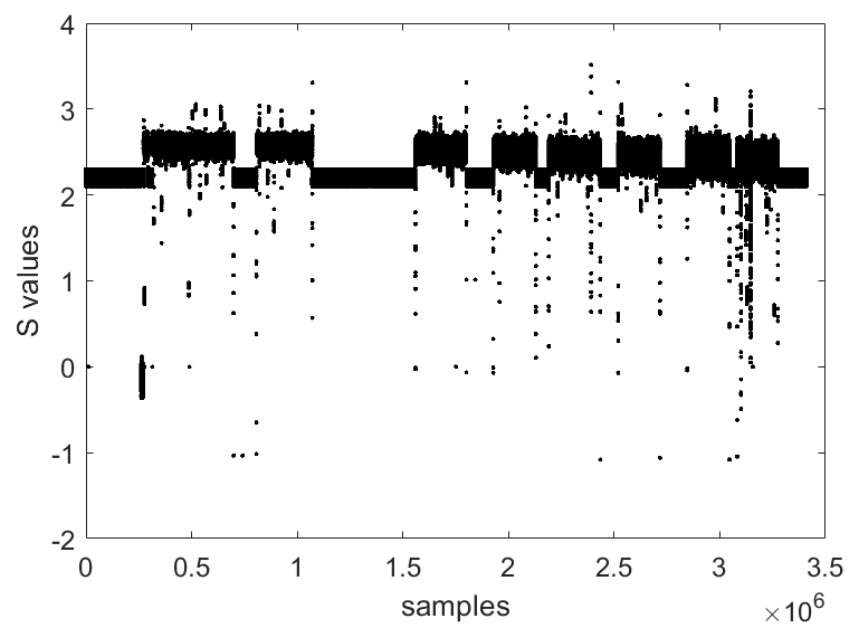

Figure 7. All collected samples in the online experiment. Values between 2.3 and 2.8 represented valid entanglement $\mathbf{S}$ measures. Values between 2.1 and 2.3 were pseudorandomly simulated samples, and outliers were quED or transmission artifacts. All pseudorandom and artifactual samples were excluded from subsequent analyses.

Figure 8 (top left) shows the data remaining after extracting the pseudo-S samples. To remove outliers, the remaining data were linearly detrended to remove long-term drift (Matlab function detrend) and then an outlier removal function (Matlab function rmoutliers) was applied with a sliding median window 10,000 samples in length. This function identifies outliers as median absolute deviations greater than 3 , based on the local median in the sliding window. These two procedures resulted in Figure 8 (top right). The final pre-processing step removed nonlinear periodicities present in the outlier-removed data. To do this, a second order SavitzkyGolay filter was applied to the data (Matlab function smooth, with option sgolay and window length 97, i.e., four epochs of 24 samples each plus 1 , the latter because the filter window length

\footnotetext{
${ }^{5}$ However, recall that part of the motivation for this experiment was to explore if mental intention could push entanglement strength above the Tsirelson bound. A subsequent article will explore in more detail whether $\mathbf{S}$ values recorded above 2.83 in this experiment were in observed vs. unobserved conditions.
} 
is required to be odd), and then the resulting smoothed curve was subtracted from the outlierremoved data. This resulted in the data shown in Figure 8 (bottom left).
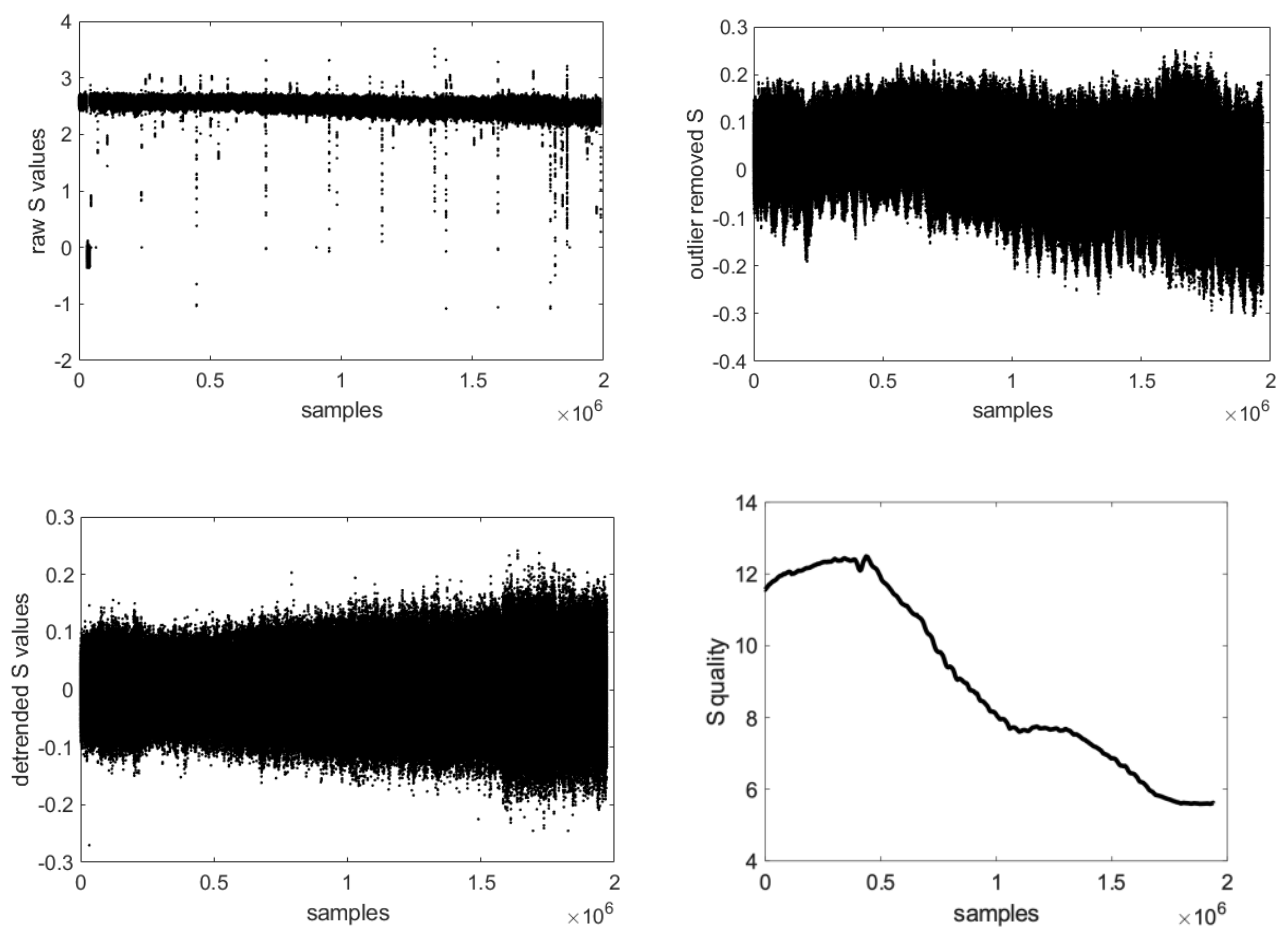

Figure 8. Top left: All $\mathbf{S}$ samples collected from the quED. Top right: After linear detrending and outlier removal. Lower left: After removal of nonlinearities with a Savitzky-Golay filter. Lower right: Photon pair entanglement quality in terms of number of sigma above classical correlations.

The increase in variance of the $\mathbf{S}$ residuals (call these $\mathbf{S}_{\mathbf{R}}$ ), which is visually evident in Figure 8 (lower left), was due to two reasons: (1) cumulative minor misalignments of the quED optics, which were in turn caused by vibrations in the polarization filters as they were rotated, and by diurnal variations in ambient temperature, and (2) degradation of electronic components in the laser's power control circuit, which reduced the laser's illumination intensity and eventually brought the experiment to a end when a key component completely failed. The consequence of this increase in variance was that the quality of the entanglement correlations progressively degraded. For the first 500,000 samples, the observed value of $\mathbf{S}$ exceeded the $\mathrm{CHSH}$ value for a classical correlation by about 12 sigma (Figure 8, lower right, calculated as sigma $\left.=\left(\mu_{\mathrm{s}}-2\right) / \sigma_{\mathrm{s}}\right)$. After 500,000 samples, entanglement quality systematically decreased, dropping to about 6 sigma by the end of the experiment. That end point was still far above the 
threshold for classical correlations, but because of the clear decrease in entanglement quality, the first 500,000 samples were analyzed separately from the remaining samples.

To evaluate the results of the experiment, each completed concentrate and relax epoch (each with 24 contiguous $\mathbf{S}$ values) was identified, and then the median of the last $8 \mathbf{S}_{\mathbf{R}}$ samples in each epoch was determined. The statistical difference in the resulting medians was evaluated using a t-test at lag 0 , this process was repeated up to lag +10 , and then the False Discovery Rate (FDR) algorithm was applied to the resulting 11 tests (Benjamini \& Hochberg, 1995).

This procedure was applied to the first 500,000 $\mathbf{S}_{\mathbf{R}}$ samples for one or more users and then compared to the same measures for zero users, which acted as a control. Then the same procedure was applied to the remaining samples. A similar analysis was then conducted for just one user, two simultaneous users, and so on, up to the maximum number of users contributing at least 20 completed concentrate and 20 relax epochs.

\subsection{Results}

The online experiment ran continuously for three months (April through June 2017). During that time, 1.97 million $\mathbf{S}$ samples were successfully received from the quED system. Of those, 228,000 $\mathbf{S}$ samples were observed by one or more participants and 1.8 million were not observed. Over 750 people participated from around the world.

Analysis of the first 500,000 samples for one or more participants identified 125,413 observed samples and 374,587 unobserved samples. Of the former, approximately 3,000 completed epochs were identified in the concentrate and relax conditions. We say "approximately" because the number of samples in the two conditions differed slightly. This occurred because in the process of removing outliers it was no longer guaranteed that a completed relax epoch would always follow a completed concentrate epoch. Likewise, when the data were lagged the attention conditions changed the boundaries of the epochs. Thus, for some epochs that had 24 contiguous samples at 0 lag, when the condition boundaries were shifted, they might no longer have had 24 contiguous samples. Any incomplete epochs were excluded from further analysis.

Comparison of the observed epochs indicated a significant increase in entanglement strength for lags 0 through 5 (after adjustment by FDR at $\mathrm{p}<0.05$, see Figure 9 , left). For the unobserved samples, some 7,800 completed epochs were identified, and none of the lags were significant. In addition, the difference in entanglement strength between observed and unobserved samples was significant for lags 0 through 7 after FDR adjustment. The same analyses applied to the remaining 1.5 million samples, of which approximately 2,200 were 
observed epochs and 28,800 were unobserved epochs, were uniformly nonsignificant after FDR adjustment (Figure 9, right).
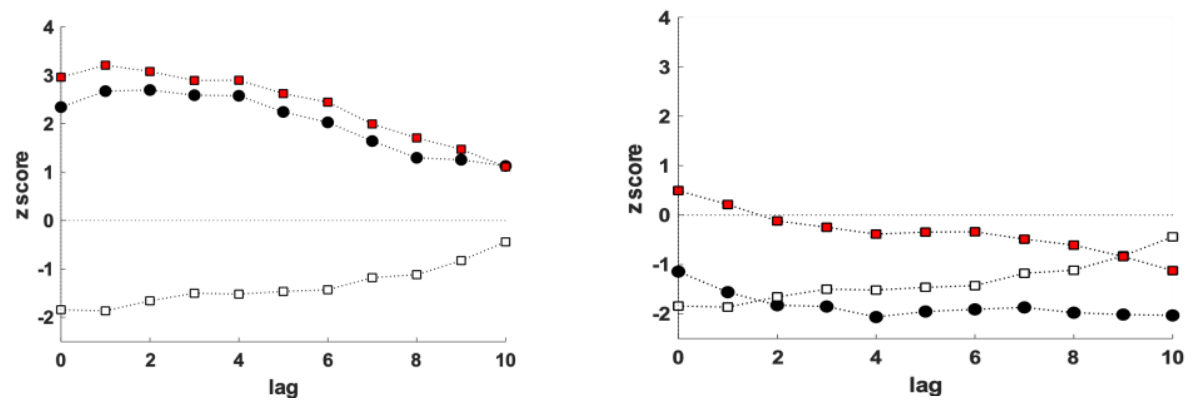

Figure 9. Black circles are results for observed data from lags 0 through 10 , white squares are the same for unobserved data, and red squares are the difference between those two curves. Left graph: Analysis of first 500,000 samples recorded during highquality entanglement. For observed data, lags 0 through 5 are significant after adjustment for False Discovery Rate; for unobserved data, no lags are significant. Right graph: Same analyses for the remaining 1.5 million samples. None of the results in either condition lags are significant.

Figure 10 shows the results by number of observers for the first 500,000 samples and for the remaining 1.5 million samples. These graphs are shown in terms of effect size rather than $z$ scores because the number of epochs contributed by multiple participants differed, as indicated by the error bars. None of these results were significant after FDR adjustment, but there is a suggestion with the initial 500,000 samples that the more simultaneous participants, the larger the resulting effect size.
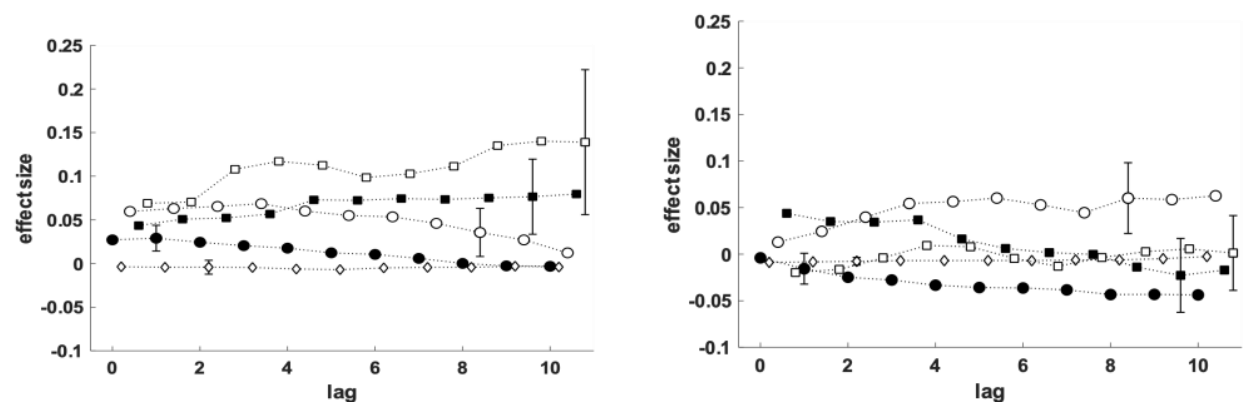

Figure 10. Black circles are experimental results in terms of effect size for one participant, white circles for two simultaneous participants, black squares for three, and white squares for four. Small diamonds represent effect size for no observers. Error bars are \pm 1 sigma. Left graph: First 500,000 samples. Right graph: Remaining 1,471,589 samples. 


\section{Discussion}

These studies probed correlations between directed mental attention and intention, versus entanglement strength in pairs of photons. To our knowledge, explorations of possible mental interactions with nonlocal forms of matter have not been previously reported. Accordingly, the results presented here should be considered preliminary. With that caveat in mind, the outcome for all four laboratory studies combined was moderately significant, at $p<0.02$, but the outcome for all three IONS experiments combined was strongly significant, at $p<0.0002$ (or possibly more significant, as this $p$-value was determined based on the number of repetitions used in the Monte Carlo procedure). Identical analyses applied to the IMI data, which were collected under different conditions and with a different participant population, did not attain significance. The online experiment produced indications of an effect on data with higher-quality entanglement, and those results may be elucidated in more detail in future analytical work.

While nonlocality is well-established as a feature of the quantum realm, its relevance and application to the mind remains unclear. These experiments were a first step in exploring whether nonlocality might play a role in this regard, or whether the seeming parallels between nonlocal mind (psi) and nonlocal matter are merely a coincidence. Should nonlocality turn out to be a common feature of both mental and quantum domains, it may provide a hint for approaches that attempt some kind of unification, such as that proposed by the philosophy of neutral monism. Progress along these lines will depend on the careful examination and subsequent extension of these first results.

A principal challenge in these studies was to devise a protocol that would accommodate unavoidable drifts in the measured strength of entanglement correlations, and the fact that the quED design required collecting coincidence counts across 16 consecutive trials to produce a single $\mathbf{S}$ value. The adopted protocol, using short, alternating epochs of concentrate and relax conditions, eliminated problems of drifts and offsets when comparing attentional conditions, but at the cost of including $\mathbf{S}$ values derived from data generated under a mix of conditions. The two analytical approaches we discussed handled this drawback by testing statistics as a function of the run lag.

The genesis of these analyses was as follows: The analysis of the online data was first devised and applied to those data and the laboratory data by the first author, using a Matlab platform. The laboratory results indicated significance exceeding the $p=0.05$ level. That analysis was then verified by the second author using new code on the Mathematica platform. At that point it became clear that an approach with more statistical power was possible, so the second author devised a new test combining three hypotheses. That procedure was developed and then applied to the data, as described herein. That analysis, however, could not be applied to 
the more complex online data. Consequently, we have reported the results of the first, simpler analysis for the online data, and a second, more detailed analysis, for the laboratory studies.

\subsection{Future studies}

These results lay the ground for future confirmatory replications in which analyses are fully specified before data collection. The studies described here provided the basis for effect size and power estimates, as well as a guide to upgrading the experimental design and apparatus. Improvements to consider are use of more stable lasers and optics, which can alleviate problems of drifts and entanglement quality, and an augmented detection system employing beam splitters instead of mirrors and 4 or 8 coincidence detectors. This would permit calculation of $\mathbf{S}$ measurements in one or two steps, instead of 16 , and allow for analyses that avoid $\mathbf{S}$-values obtained under mixed attentional conditions.

A deficiency of the lab experiments, which became clear in hindsight, is that they did not collect baseline data immediately before and after each session. Baselines of 5 to 10 minutes immediately prior to and after data collection (with participants absent and without any adjustments to the apparatus) would be very helpful in documenting the equipment's stability. This is an obvious feature to be included in future studies.

\subsection{Challenges}

Before turning to the novel implications of these experiments, we address several wellknown issues in research involving psi phenomena. The first is the variability and difficulty in reproducing psi effects (Rao, 1985). Obtaining different results in seemingly comparable circumstances, as we found for the IONS and IMI lab studies, is not uncommon in this domain, or indeed in many other, more conventional experimental domains (Hudson, 2021). Discussions about a "reproducibility crisis" in science remain an ongoing challenge in identifying and firmly establishing all sorts of interesting effects, including psi (Guttinger, 2020).

Still, we expect that process-oriented psi research will continue to make progress. Two recognized factors for obtaining better results are the use of participants who performed well on previous studies, as well as a setting that allows for the participants to apply their full and relaxed attention to the experimental tasks. While the IONS experiments attempted to optimize these factors, this was not the case for the IMI study. The IMI experiment was conducted with firsttime participants who had limited time to settle into the experimental sessions because they were following the demanding schedule of a group meditation retreat. Another consideration was the relatively small size of the participant pool at IONS, which can impact the variability of effect 
sizes. The discrepancy in results is thus not inconsistent with previous experience and observations reported in the psi literature.

A second issue pertains to the problem of determining the locus of the effect, as alluded to in the Introduction. Two competing interpretations of psi effects are the psychokinetic (PK) paradigm (Varvoglis \& Bancel, 2015), and that of precognitive selection (May et al., 1995). Under PK, attention and/or intention are assumed to not only correlate with measured deviations in the behavior of the physical system, but to cause those changes. By contrast, precognitive selection posits that the participant's or the investigator's intuition informs the fortuitous timing of data collection so as to take advantage of naturally occurring fluctuations in the data which "just happen to" favor confirmation of the hypothesis.

Both ideas assume the existence of anomalous psi effects, but with the selection paradigm the target system is not influenced at all, only a biased selection of data are extracted from an otherwise unperturbed system. The analyses we used in the experiments described here could not definitively distinguish between these two mechanisms, and we would have detected the same statistical results in either case. However, because we are ultimately interested in inferences that might be drawn from a causal PK effect on entanglement, this was a limitation in the current experiment's design, which should be addressed in future studies.

Ways to potentially control for selection effects include reducing the degrees of freedom for when the experimenter or participant decides to start a session, collecting data before and after sessions, and randomizing session durations. These measures should be understood through modeling and folded into future protocols. Although the preliminary protocol reported here did not consider this design feature, the results do provide some peripheral support of the PK hypothesis because of the nonsignificant result of the feedback hypothesis, H4. A straightforward interpretation of the selection hypothesis would have predicted that the direction of observed effects and the method of feedback should agree, whereas there was no evidence of that in these data. We will be interested to see if this speculation is borne out by a future protocol that incorporates selection screening.

If such a protocol were devised, and the results were consistent with a PK effect, then it is still not certain that the results could be attributable only to a modulation of entanglement strength. Alternatives could include influences on elements of the optical apparatus, the electronics of the coincidence counter, or some other component of the experimental system. That is, a PK effect might take advantage of well-known weaknesses (loopholes) within the standard Bell-CHSH experiment (Yang \& Zhang, 2021). Consequently, identifying and closing these loopholes will be necessary to strengthen evidence for a PK effect that specifically modulates entanglement. A full discussion of this issue is beyond the scope of this paper, but two 
possible avenues worth mentioning are (a) to adapt solutions used to close standard loopholes in Bell experiments, particularly the so-called detection and measurement independence (freechoice) loopholes which can be viewed (under the psi hypothesis) as a hybrid of PK and selection; and (b) to explore other types of entanglement experiments, such as quantum steering or tripartite scenarios that might offer independent support for a true entanglement effect (Ruzbehani, 2021).

\subsection{Implications}

Assuming for the moment that the mind-modulated entanglement hypothesis is correct, several observations and potential inferences follow. Our results are the first to address quantum nonlocality directly as a potentially deep, albeit ill-defined connection between psi phenomena and quantum systems. That is, previous mind-matter interaction experiments have involved psi interactions with distant physical systems, but not physical systems known to have nonlocal properties. That includes previous experiments designed to test if focused attention and/or intention can modulate quantum-based randomness, such as electron tunneling phenomena in semiconductors (Jahn et al., 2007), and also experiments testing whether psi perception can influence photon interference in a double-slit optical system (Radin et al., 2021).

A second observation is that, lacking evidence for intentional steering (as per H4), it may be that observed effects on entanglement strength are associated with mere attention alone. This would stand in contrast with the relevant literature on so-called goal-oriented psi effects, in particular the results of many micro-PK studies using truly random number generator outputs as targets (Bancel, 2017; Varvoglis \& Bancel, 2015). Many of those studies conclude that intention "steered" the direction of the effect, contrary to what we observed in these experiments. What is less clear is whether those previous studies are explainable in terms of psi selection (May et al., 1995), or instead suggestive of PK (Dobyns, 2000). Finding that the distinction between intentional steering and the application of mere attention is associated with a PK/psi-selection dichotomy would be a useful advance that could influence theoretical models as well as advances in experimental design.

The measured increase in $\mathbf{\Delta S}$ suggests that the quality of entanglement (and by extension its value as a nonlocal resource) is increased when coupled to attentional awareness. This is surprising because interactions with external systems generally cause entanglement to dissipate. There exist quantum information procedures for increasing the quality (fidelity) of entanglement, but these "distillation" protocols involve operations on multiple copies of entangled qubits (Ecker et al., 2021), and it is not obvious how that is relevant to coincidence measurements in single photon pairs, as measured in our experiments. Further investigations of quantum distillation might provide insights into how entanglement could be accessible to processes associated with 
mental states. Theoretical questions aside, it is important to note that any process that increases the fidelity of entangled states is of central interest in quantum information technologies such as computing, encryption, key distribution, and teleportation. That consideration alone provides a strong motivation for continued research.

The magnitude of the $\mathbf{\Delta S}$ increase that we measured is also noteworthy. We found $\boldsymbol{\Delta} \mathbf{S}_{\mathbf{e x p}}$ $\approx 0.0057 \pm 0.0024$. The most refined attempts to date to approach the upper limit of quantum entanglement (the Tsirelson Bound) have reported values of $\mathbf{S}_{\mathbf{T B}}-\mathbf{S}$ as small as $0.0008 \pm 0.0005$ (Poh et al., 2015). The size of the positive modulations in $\mathbf{S}$ that we measured thus exceeds the gap between the Tsirelson Bound and the best reported $\mathbf{S}$-values. Our exploratory experiments are thus successful in demonstrating that a test to see if mind can modulate entanglement beyond the Tsirelson Bound is a practicable goal. Such a test, if successful, would have deep implications. If the Tsirelson Bound is in fact a hard limit for psi-mediated entanglement, as it is for conventionally prepared quantum systems, that would indicate that psi phenomena obey quantum theory and suggest that theories of psi should be compatible with current physics, at least as far as information dynamics are concerned. However, if mental intention violates the Tsirelson Bound, it would imply that quantum theory provides an incomplete description of the world when systems incorporate certain mental phenomena. Such a test could empirically inform the metaphysical puzzle that seeks to discover the fundamental constituents of our world. In that case, a suitably devised test might be able to distinguish qualia as more fundamental than the qubit, or vice versa. In philosophical terms, this could be a test of idealism versus materialism, or it could offer guidance for the formulation of a "middle way," such as neutral monism.

As this discussion makes clear, there is much to do before such an experiment can be performed in a convincing manner. It is worth mentioning that it took nearly 40 years to achieve a loophole-free demonstration of nonlocal Bell correlations. ${ }^{6}$ The first step toward such a test in the present domain would require access to entanglement sources with improved stability and fidelity. Then it would be useful to design optical configurations with multiple coincidence detectors and fixed analyzers, as that would eliminate the need for stepping through the 16 polarization conditions, providing single-shot measurements of $\mathbf{S}$. Such a set-up would provide

\footnotetext{
${ }^{6}$ That is, experiments that simultaneously close the locality and detection loopholes. Very strong constraints have been put on the so-called free-choice (or, measurement independence) loophole. But this loophole cannot be closed completely. A demonstration of Bell nonlocality that simultaneously addresses all three has yet to be performed.
} 
further flexibility for designing comparisons between concentrate and relax periods, and it would facilitate experiments to explore the key question of whether the underlying effect is better understood as PK or as selection. Equally important would be attracting interest from researchers in the quantum information community, who could supply much-needed advice on a range of subtle issues regarding entanglement. We hope that this paper will serve in that regard.

\subsection{Replication Issues}

On the psychological side of the hypothesized psychophysical effect, it is important to emphasize that the experiments described here, as well as possible future designs, are not simply physics experiments. It would be a mistake to assume that simply asking a person to focus their attention toward the physical apparatus will be sufficient to observe the effect. The phenomenon at issue here is more subtle, involving psychological attributes of the experimental set and setting, as well as tacit factors that have yet to be fully recognized or elaborated.

As already mentioned, among factors suspected to influence outcomes in previous psi experiments, there are obvious variables like the participant's level of comfort with the environment in which the test is conducted. In addition, the type and presentation of specific tasks involved, the nature of the feedback (if any), the outward attitudes of the experimenters (e.g., warm and enthusiastic versus cold and skeptical), and unconscious biases or concerns held by either participants or investigators, may also influence outcomes.

Another consideration is how best to match participants to a given experimental protocol. The assumption that all participants are equal is obviously an error, and psi research has shown that results are improved when some pre-selection of participants is adopted (Alexander et al., 1998; Ryzl, 1963). However, the strategies of participant selection are more of an art than a science at this point, and as such it adds overhead to the experimental program.

With such a range of factors that might influence the outcome, developing replicable experimental protocols will likely take substantial resources. Our sense is that a long-term view is needed, one that can support a program of research that progressively addresses the full range of experimental and protocol issues outlined above. As this preliminary report suggests, such a program appears to be achievable, and it is certainly justified given the fundamental nature of the questions addressed.

\section{Author Contributions}

Dean Radin secured funding for this project, conducted three laboratory and online experiments, analyzed the data, and prepared the first draft of the manuscript. Peter Bancel 
conducted one lab study, confirmed the initial analyses, developed a more refined approach to analyze the laboratory studies, and contributed significantly to subsequent drafts of the manuscript. Arnaud Delorme designed and developed the computing infrastructure, the software used to collect data in both the lab and online studies, and he reviewed the draft manuscript.

\section{Declaration of competing interests}

The authors declare no conflicts of interest.

\section{Acknowledgements}

We are grateful to the Emerald Gate Charitable Trust, the Bial Foundation, the John B. Huntington Foundation, the Hittman Family Foundation, Richard and Connie Adams, Jeff Parrett, Patrick Dewavrin, and the donors and members of the Institute of Noetic Sciences and the Institut Métapsychique International for their generous support.

\section{References}

Aspect, A., Dalibard, J., \& Roger, G. (1982). Experimental test of Bell's inequalities using time-varying analyzers. Physical Review Letters, 49(25), 1804-1807. https://doi.org/10.1103/PhysRevLett.49.1804

Benjamini, Y., \& Hochberg, Y. (1995). Controlling the False Discovery Rate: A practical and powerful approach to multiple testing. Journal of the Royal Statistical Society. Series B (Methodological), 57(1), 289-300. https://doi.org/10.2307/2346101

Brunner, N., Cavalcanti, D., Pironio, S., Scarani, V., \& Wehner, S. (2014). Bell nonlocality. Reviews of Modern Physics, 86(2), 419-478. https://doi.org/10.1103/RevModPhys.86.419

Bub, J. (2019). Quantum entanglement and information. In E. N. Zalta (Ed.), The Stanford Encyclopedia of Philosophy (Spring 2019). Metaphysics Research Lab, Stanford University. https://plato.stanford.edu/archives/spr2019/entries/qt-entangle/

Cardeña, E. (2015). Parapsychology: A Handbook for the 21st Century (J. Palmer \& D. Marcusson-Clavertz, Eds.). McFarland.

Cardeña, E. (2018). The experimental evidence for parapsychological phenomena: A review. American Psychologist, 73(5), 663-677. https://doi.org/10.1037/amp0000236 
Chiribella, G., \& Spekkens, R. W. (Eds.). (2015). Quantum Theory: Informational Foundations and Foils (1st ed. 2016 edition). Springer.

Clauser, J. F., Horne, M. A., Shimony, A., \& Holt, R. A. (1969). Proposed experiment to test local hidden-variable theories. Physical Review Letters, 23(15), 880-884. https://doi.org/10.1103/PhysRevLett.23.880

Corneille, J. S., \& Luke, D. (2021). Spontaneous spiritual awakenings: Phenomenology, altered states, individual differences, and well-being. Frontiers in Psychology, 12. https://doi.org/10.3389/fpsyg.2021.720579

Correa, R., Rodriguez, N., \& Bortolaso, M. (2022). What is the nature of the alteration of temporality in trauma-related altered states of consciousness? A neurophenomenological analysis. European Journal of Trauma and Dissociation, 6(2). https://doi.org/10.1016/j.ejtd.2021.100227

Cuffaro, M. E. (2018). Information causality, the Tsirelson bound, and the 'being-thus' of things. Studies in History and Philosophy of Science Part B: Studies in History and Philosophy of Modern Physics. https://doi.org/10.1016/j.shpsb.2018.05.001

Ecker, S., Sohr, P., Bulla, L., Huber, M., Bohmann, M., \& Ursin, R. (2021). Experimental singlecopy entanglement distillation. Physical Review Letters, 127(4). https://doi.org/10.1103/PhysRevLett.127.040506

Gopher, D., Greenshpan, Y., \& Armony, L. (1996). Switching attention between tasks: Exploration of the components of executive control and their development with training. Proceedings of the Human Factors and Ergonomics Society Annual Meeting, 40(21), 1060-1064. https://doi.org/10.1177/154193129604002103

Guttinger, S. (2020). The limits of replicability. European Journal for Philosophy of Science, 10(2). https://doi.org/10.1007/s13194-019-0269-1

Hudson, R. (2021). Should we strive to make science bias-free? A philosophical assessment of the reproducibility crisis. Journal for General Philosophy of Science, 52(3), 389405. https://doi.org/10.1007/s10838-020-09548-w

Jahn, R. G., Dunne, B. J., Nelson, R. G., Dobyns, Y. H., \& Bradish, G. J. (2007). Correlations of random binary sequences with pre-stated operator intention: A review of a 12-year program. Explore (New York, N.Y.), 3(3), 244-253, 341-343. https://doi.org/10.1016/j.explore.2007.03.009

Kennedy, J. E. and T. J. L. (1976). Experimenter effects in parapsychological research ***. Journal of Parapsychology, 40(1), 1-33. 
May, E., Utts, J., \& Spottiswoode, S. J. P. (1995). Decision augmentation theory: Toward a model of anomalous mental phenomena. Journal of Parapsychology, 59, 195-220.

Mendoza-Martínez, M. L., Vallejo, J. A., \& Zúñiga-Galindo, W. A. (2019). Acausal quantum theory for non-Archimedean scalar fields. Reviews in Mathematical Physics, 31(4). https://doi.org/10.1142/S0129055X19500119

Mossbridge, J., \& Radin, D. (2018). Precognition as a form of prospection: A review of the evidence. Psychology of Consciousness: Theory, Research, and Practice, 5(1), 78.

Musser, G. (2018). What is spacetime? Nature, 557(7704), S3-S6. https://doi.org/10.1038/d41586-018-05095-z

Radin, D., Wahbeh, H., Michel, L., \& Delorme, A. (2021). Psychophysical interactions with a double-slit interference pattern: Exploratory evidence of a causal influence. Physics Essays, 34, 79-88.

Rao, K. R. (1985). Replication in conventional and controversial sciences. In Betty Shapin and Lisette Coly (Eds.), The Repeatability Problem in Parapsychology: Proceedings of an International Con- Ference Held in San Antonio, Texas, October 28-29, 1983 (Pp. 22-34; Discussion: Pp. 34-41). New York: Parapsychology Foundation, 1985.

Ruzbehani, M. (2021). Simulation of the Bell inequality violation based on quantum steering concept. Scientific Reports, 11(1). https://doi.org/10.1038/s41598-02184438-9

Schmidt, H. (1987). The strange properties of psychokinesis. Journal of Scientific Exploration, 1(2), 103-118.

Schooler, J. W., Baumgart, S. L., \& Franklin, M. (2018). Entertaining without endorsing: The case for scientific investigation of anomalous cognition. Psychology of Consciousness: Theory, Research, and Practice, 5(1), 63-77.

Schrödinger, E. (1935). Discussion of probability relations between separated systems. Mathematical Proceedings of the Cambridge Philosophical Society, 31(4), 555-563. https://doi.org/10.1017/S0305004100013554

Schwartz, S. (2015). Through time and space: The evidence for remote viewing. In The evidence for psi. Eds. D. Broderick and B. Groetzel. McFarland: New York.

Sheehan, D. P. (2006). Frontiers of time: Retrocausation experiment and theory. In AIP Conference Proceedings 863. American Institute of Physics.

Stubenberg, L. (2018). Neutral Monism. In E. N. Zalta (Ed.), The Stanford Encyclopedia of Philosophy (Fall 2018). Metaphysics Research Lab, Stanford University. https://plato.stanford.edu/archives/fall2018/entries/neutral-monism/ 
Svozil, K. (2018). Physical (1st ed. 2018 edition). Springer.

Tressoldi, P. E., \& Storm, L. (2021). Stage 2 Registered Report: Anomalous perception in a Ganzfeld condition - A meta-analysis of more than 40 years investigation (10:234). F1000Research. https://doi.org/10.12688/f1000research.51746.1

Velmans, M. (2017). Towards a deeper understanding of consciousness: Selected works of Max Velmans (pp. vii, 233). Routledge/Taylor \& Francis Group.

Yang, S., \& Zhang, S. (2021). Loophole-free Bell test with multi-photon-subtracted twomode squeezed state. Optik, 231. https://doi.org/10.1016/j.ijleo.2021.166261

\section{Appendices}

\section{A. Calculation of S-values and $\Delta S$.}

The following procedure generates an $\mathbf{S}$-value for any combination of experimental sessions. Data trials are extracted using selection windows of 16 consecutive trials (one trial for each of the 16 analyzer combinations). Windows are placed at identical locations within runs, which assures that they have identical SCC assignments (see section C). The trials are then sorted by polarization setting and summed to the yield the total coincidence counts for each setting. For polarizers in the two wings ${ }^{7},(\mathbf{A}, \mathbf{B})$, of the experiment, and with orientations $\mathbf{a}$ and b, the correlation coefficient is given as :

$$
E(\boldsymbol{a}, \boldsymbol{b})=\frac{N_{++}(\boldsymbol{a}, \boldsymbol{b})-N_{-+}(\boldsymbol{a}, \boldsymbol{b})-N_{+-}(\boldsymbol{a}, \boldsymbol{b})+N_{--}(\boldsymbol{a}, \boldsymbol{b})}{N_{++}(\boldsymbol{a}, \boldsymbol{b})+N_{-+}(\boldsymbol{a}, \boldsymbol{b})+N_{+-}(\boldsymbol{a}, \boldsymbol{b})+N_{--}(\boldsymbol{a}, \boldsymbol{b})},
$$

where \pm refers to a polarization analyzer setting parallel or perpendicular to orientations $(\boldsymbol{a}, \boldsymbol{b})$. The CHSH scenario measures correlations for two different polarizer orientations in each wing, A and B. With these orientations denoted as $\left(\boldsymbol{a}, \boldsymbol{a}^{\prime}\right)$ and $\left(\boldsymbol{b}, \boldsymbol{b}^{\prime}\right)$, the S-value is given by:

$$
\boldsymbol{S}_{\text {exp }}=E(\boldsymbol{a}, \boldsymbol{b})-E\left(\boldsymbol{a}, \boldsymbol{b}^{\prime}\right)+E\left(\boldsymbol{a}^{\prime}, \boldsymbol{b}\right)+E\left(\boldsymbol{a}^{\prime}, \boldsymbol{b}^{\prime}\right) .
$$

The settings for our experiment are the CHSH settings that maximize $\mathbf{S}:\left(\boldsymbol{a}, \boldsymbol{a}^{\prime}\right)=\left(0^{\circ}, 45^{\circ}\right)$ and

${ }^{7}$ The 'wings' are the flight paths of the two entangled photons. Each wing is similarly configured with a polarization analyzer, mirrors, and fiber optics that direct the photon to a coincidence detector. 
$\left(\boldsymbol{b}, \boldsymbol{b}^{\prime}\right)=\left(22.5^{\circ}, 67.5^{\circ}\right)$. The standard deviation of $S_{\text {exp }}$ can be derived with the assumption that the counting error for $\mathrm{n}$ counts is $\sigma_{n}=\sqrt{n}$, whereby from propagation of errors, we have:

$$
\sigma_{S}=\sqrt{\sum_{i=1}^{16} N_{i}\left(\frac{\partial S}{\partial N_{i}}\right)^{2}} .
$$

The differential values, $\Delta \mathbf{S}$, that are designated by the hypotheses are given by:

$$
\Delta S=S_{L}-S_{L+27},
$$

where $\mathrm{L}$ is a lag, $\mathrm{L}=0$ positions the selection window at the last of the concentrate trials, and $\mathrm{L}+27$ is a lag shifted by half of a run-length from $\mathrm{L}$ (the lag is $\mathrm{L}+31$ for IMI data). The value $\Delta \mathbf{S}$ constitutes the effect size for our experiments. For our choice of a 16-trial window for TCC, SCC has its maximum at $\mathrm{L}=2.5$ for IONS data and $\mathrm{L}=6$ for IMI data, so for the respective hypothesis tests $\mathrm{L}=(2.5,6)$ is used. The lag of the $\mathrm{SCC}$ maximum is moderately sensitive to changes in the TCC window: \pm 2 trials in TCC window length produce a lag change in SCC of \pm 1. Because $\boldsymbol{S}_{\boldsymbol{L}}$ and $\boldsymbol{S}_{\boldsymbol{L}+27}$ derive from nearly equivalent quantities of data, the standard error of $\Delta \mathbf{S}$ is taken as:

$$
\sigma_{\Delta S}=\sigma_{S} \sqrt{2} .
$$

It is important to note that combining data from different sessions will not lead to accidental biases of $\mathbf{\Delta} \mathbf{S}$ because coincidence data from any given window at a lag $\mathrm{L}$ is always paired with nearby data in the same run at L+27. Any possible shifts in the baseline levels of $\mathbf{S}$, which occur as the laser output and optical alignments change from session to session, will cancel out when the paired $\mathbf{S}$-values at lags $\mathrm{L}$ and $\mathrm{L}+27$ are subtracted. In addition, calibrations show that biases of $\mathbf{\Delta S}$ due to drifts within a session are below the level of detectability for our data.

\section{B. Fits of the $\Delta S$ lag curve.}

The $\Delta \mathbf{S}$ lag curve is calculated from lags 0 to 503 , as described in the text. The following values are calculated for each lag, $\mathrm{L}_{\mathrm{m}}$ :

- The $\mathbf{S}$-value of the 16-trial window terminating at $\mathrm{L}_{\mathrm{m}}$.

- The $\mathbf{S}$-value of the window displaced by $1 / 2$ a run, at lag $\mathrm{L}_{\mathrm{m}}+27$ (for IONS data; the displacement is 31 for the IMI data). 
- The difference of the two $\mathbf{S}$-values, $\Delta \mathbf{S}$.

- The Poisson statistical errors of these 3 values.

- The number of (Poisson) standard deviations from zero of $\mathbf{\Delta S}$ (the z-score).

The $\mathbf{\Delta S}$ lag curve is then fit to the Cosine function,

$$
\Delta S_{L}=A * \operatorname{Cos}\left(\frac{2 \pi}{L_{R}} L-\phi\right)
$$

where the amplitude (taken as $\mathrm{A} \geq 0$ ) and phase are free fit parameters, and $\mathrm{L}$ is the lag. The frequency $2 \pi / L_{R}$ is fixed and corresponds to run lengths of 53 (61 for IMI data) lags for the IONS(IMI) data, taking a value of $0.118551(0.103003)$. The fit is done using the NonlinearModelFit function of Mathematica (version 12.1). The test statistic for H1, MaxZ, is taken as the absolute value of the extremum of the $\Delta \mathbf{S}$ lag curve. The values of $\mathbf{A}$ and $\phi$ are the best fit parameters to the curve.

\section{Determination of test lag for Hypothesis 3}

The overall prediction, based on the hypothesis that mind can directly influence aspects of distant material systems, was that deviations in $\mathbf{S}$-values would be associated with the participants 'intentional focus during the concentrate epochs as compared to the relax epochs. One complication for analysis was that the measurements of $\mathbf{S}$ were calculated over 16 consecutive trials, which meant that data from a previous instructional condition could overlap with the present condition. This in turn meant that the hypothetical mental influences on $\mathbf{S}$ would not appear instantaneously when the computer announced the next condition, but rather it would become increasingly evident with some time lag. This lag would affect how the $\mathbf{S}$-value of a given trial should be associated with a given condition.

In addition, it was expected that participants would need time to cognitively "switch gears" when shifting their attention between successive conditions (Gopher et al., 1996). This too would affect how $\mathbf{S}$-values and conditions should be associated because participants ' effectiveness in focusing their attention might possibly change over the duration of each epoch as the session proceeded.

Our approach toward addressing these issues was to construct an "S-value Condition Coefficient" (SCC). The SCC provided a way to analyze any collection of trials used to calculate $\mathbf{S}$ and to quantify the degree to which the data were expected to exhibit lagged "concentrate-like" or "relax-like" behavior. The construction of SCC is discussed below. 


\section{S-value Condition Coefficients (SCC):}

A SCC coefficient is assigned to each trial. As a first step, a Trial Condition Coefficient (TCC) is constructed. The TCC quantifies the induction of a participant's momentary psi effort at the time of a trial. TCC is defined as the average of instructional conditions across a window of preceding trials. It thus represents the immediate history of intentional efforts and is motivated by the assumption that a brief time is needed for participants to adapt and "settle into" each condition. For example, a psi effect might increase over the duration of an instructional epoch because a participant has time to settle into the condition as trials proceed. A "history" window of 16 consecutive trials is used for TCC, which corresponds in these experiments to about 20 seconds. This corresponds to 3-4 slow breaths and is long enough for participants to process cognitively and to fully sense the change between conditions, yet it is shorter than the 24-trial epochs of each condition. By designating values of 0,1 and -1 to the conditions get ready, concentrate and relax, the TCC obtains values between \pm 1 .

The construction of the $\mathbf{S}$-value Condition Coefficient (SCC) is taken as the average of the TCCs over the 16-trial S-value calculation window. The SCC is thus a weighted average of the underlying condition parameters $(-1,0,1)$ for the 31 trials preceding an $\mathbf{S}$-value. The TCC and $\mathrm{SCC}$ for the experiments, and their relation to the run structure are shown in Figure A.1. Finally, the SCC for $\mathbf{\Delta S}$ is simply the difference of two SCC's, lagged by 27 (or 31, for IMI data) trials ${ }^{8}$.

${ }^{8}$ The 16-trial window for constructing SCC was dictated by the experimental setup and the $\mathbf{C H S H}$ calculation for $\mathbf{S}$. However, the choice of a 16-trial window for the TCC was based on psychological considerations. Nevertheless, SCC is not too sensitive to the TCC window choice, and its period is the run length, regardless of that choice. 


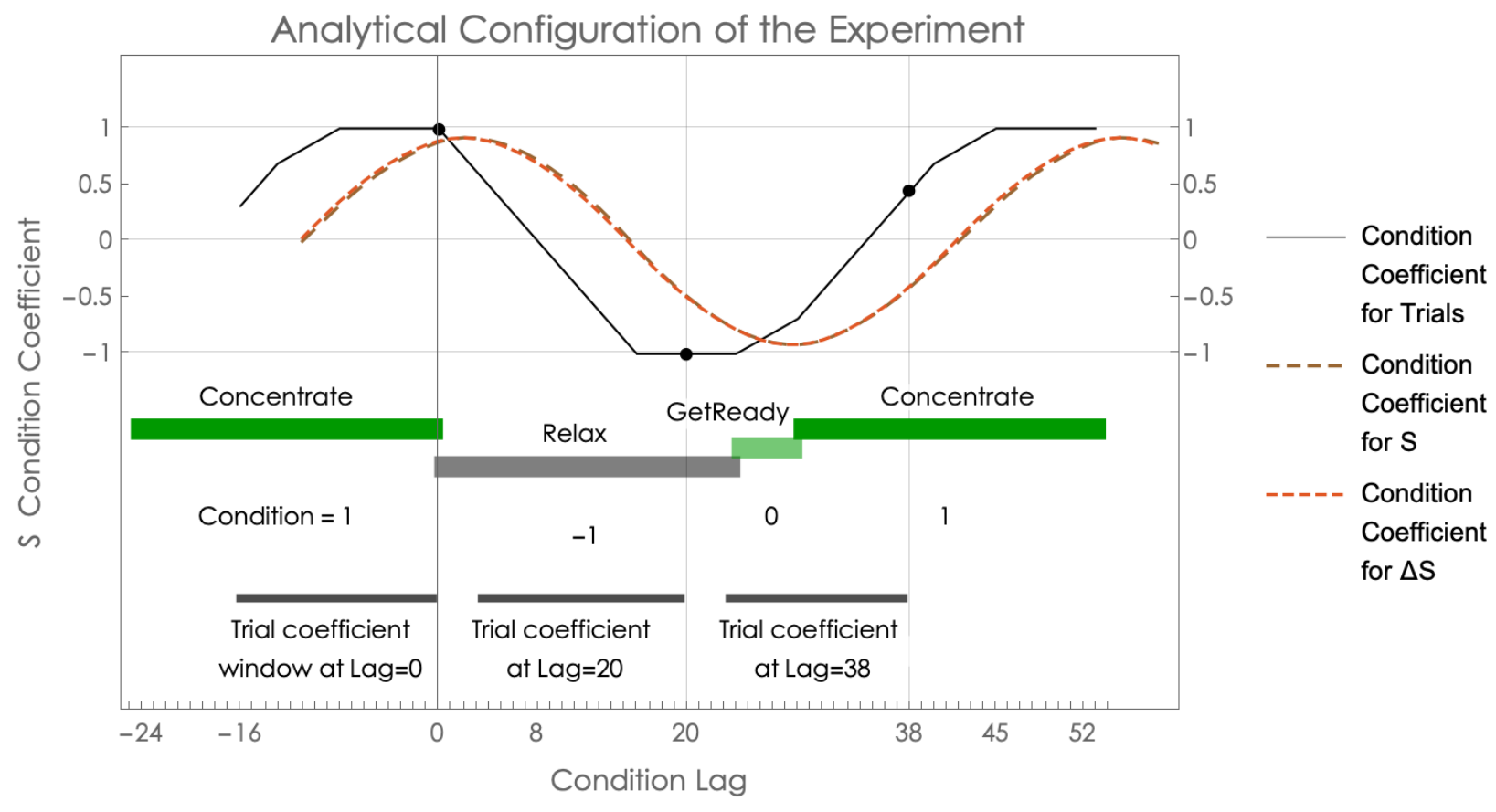

Figure A.1. Construction of $\mathbf{S}$ Condition Coefficients (SCC) for the experiment. The figure shows the value of condition parameters $(0,1,-1)$, the Trial Condition Coefficient (TCC), and SCC within an experimental run. Trials are assigned lags with respect to lag $=0$ for the last concentrate trial. The thick horizontal bars (in green and gray) indicate the position and durations of the get ready, concentrate and relax instructions, relative to lag 0 . Narrow horizontal bars (in black) are 16-trial selection windows for calculating S, TCC and SCC. They are positioned at lags 0,20 and 38 in this example. These windows select which trials are used for calculations of $\mathbf{S}$. The selected trial data may be coincidence counts when calculating $\mathbf{S}$, the condition parameters when calculating TCC, or the TCCs when calculating SCC. All of these quantities are indexed by the window's lag. The TCC values for these windows are indicated by the black points on the TCC curve. The maximum of SCC occurs at lag $=2.5$, which gives the lag positions for $\mathbf{S}$-values to test hypotheses 1 and 3.

We note the following about lagged curves for $\mathbf{S}, \mathbf{\Delta S}$ and SCC. Because hypotheses 2 and 3 assume that an effect has a modulation period equal to the run length $L_{R}$, tests can be performed by fitting $\Delta \mathbf{S}$ to a periodic function. Note that even if an effect is absent, lagged curves can be approximately cyclic with a period $L_{R}$. This is because values of $\Delta \mathbf{S}$ lagged modulo $L_{R}$ use subsets of the same data. That is, if the run length is $L_{R}$, and if $\left\{\Delta \mathbf{S}_{\mathbf{n}}\right\}$ are taken at intervals $L+n \cdot L_{R},(n=1,2, \ldots)$, then $\left\{\Delta \mathbf{S}_{\mathbf{n}}\right\}$ share subsets of the data for $\boldsymbol{\Delta} \mathbf{S}_{\mathbf{0}}$. Curves lagged up to $\mathrm{L}_{\mathrm{R}}$ effectively show the evolution of $\boldsymbol{\Delta} \mathbf{S}$ over the course of an average run. For lags extending beyond $L_{R}$, the $\Delta \mathbf{S}$ curve provides information on how the run profile changes during a session. 
Lagged curves are thus useful for determining the amplitude and phase of data structure relative to the run length, and for displaying the evolution of effects over the longer timescale of a full session.

\section{Monte Carlo estimations of test $p$-values.}

The Null distribution of MaxZ for the test of H1 is built out of Monte Carlo calculations of the lag curve and the distribution of $\mathbf{A}$ is estimated from Cosine fits of the Monte Carlo lag curves. The surrogate data are generated as follows. For each experimental session, the average of coincidence counts at each polarization setting is calculated. This yields a matrix $\mathbf{M}_{\mathbf{p s}}$ where $\mathbf{p}$ indexes the 16 polarization settings, and $\mathbf{s}$ indexes the session. $\mathbf{M}_{\mathbf{p s}}$ thus reproduces the average levels of coincidence counts measured in the experiment, for all sessions and analyzer settings. To generate a surrogate Null dataset, the experimental coincidence counts for each trial are replaced with random variables drawn from Poisson distributions with mean parameters given by $\mathbf{M}_{\text {ps. }}$. The result is a simulation under the assumption of the Null hypothesis, and with the same structure and count levels as the experimental data. Test statistic $p$-values are estimated by counting the fraction of test values calculated for the Monte Carlo surrogate datasets that exceed the experimental values, MaxZ and A (see Figure A.2). Distribution estimates typically used 4,000 Monte Carlo iterations.

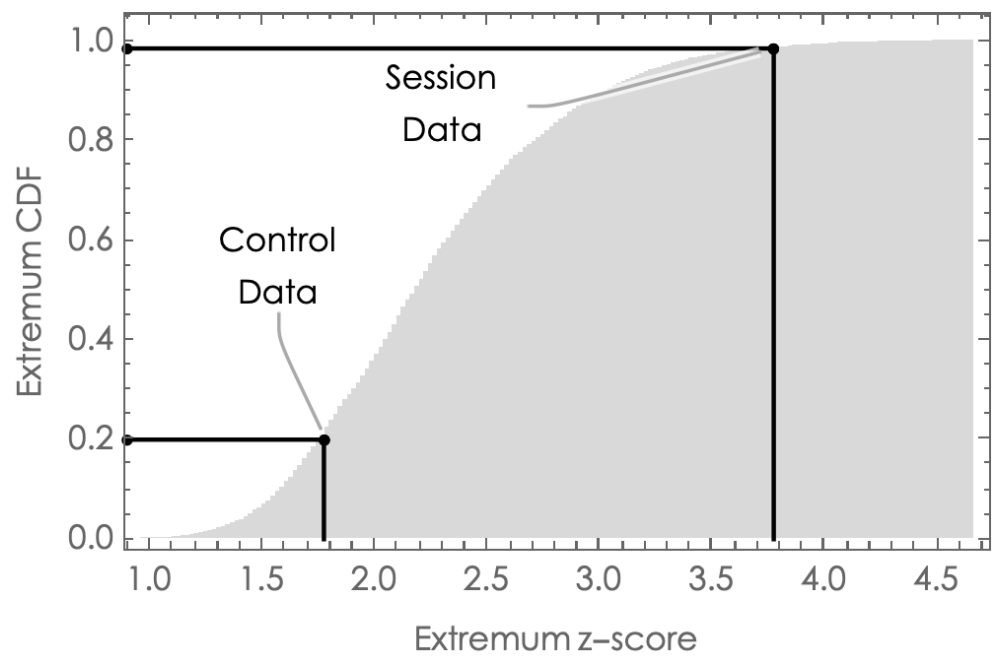

Figure A.2. The results and Monte Carlo CDF for the test of H1. The horizontal axis is the extremum z-score of the $\Delta \mathbf{S}$ lag curve for 4000 Monte Carlo simulations. The vertical axis gives the cumulative probability. The $p$-value of MaxZ for the IONS data is $p=0.003$ ( $p$-values for the extrema are 1-tailed and are given by 1-CDF). 
The Cosine fits also yield a Null distribution for $\phi$. However, since H3 tests both the value and the variability of this quantity, an estimate of the Null distribution of the standard deviation of $\phi$ is also needed. The distribution for $\sigma_{\phi}$ is estimated by a bootstrap analysis, as follows. From each surrogate Null dataset, a new dataset is generated by random sampling of the sessions, with replacement. A Cosine fit of the resampled data yields a new value for $\phi$. The procedure is repeated on the initial surrogate 100 times and the standard deviation of the $\phi$ 's is calculated. The estimated distribution of $\sigma_{\phi}$ is obtained by repeating the resampling analysis for each one of the (4000) surrogate Monte Carlo datasets (see Figure A.3). Note that grouped values of MaxZ, $\mathbf{A}$, and $\sigma_{\phi}$ are identified with each individual Monte Carlo dataset. This will be important for treating dependencies among tests in the determination of the Null distribution of $F c h i$, the Fisher combination of $p$-values.

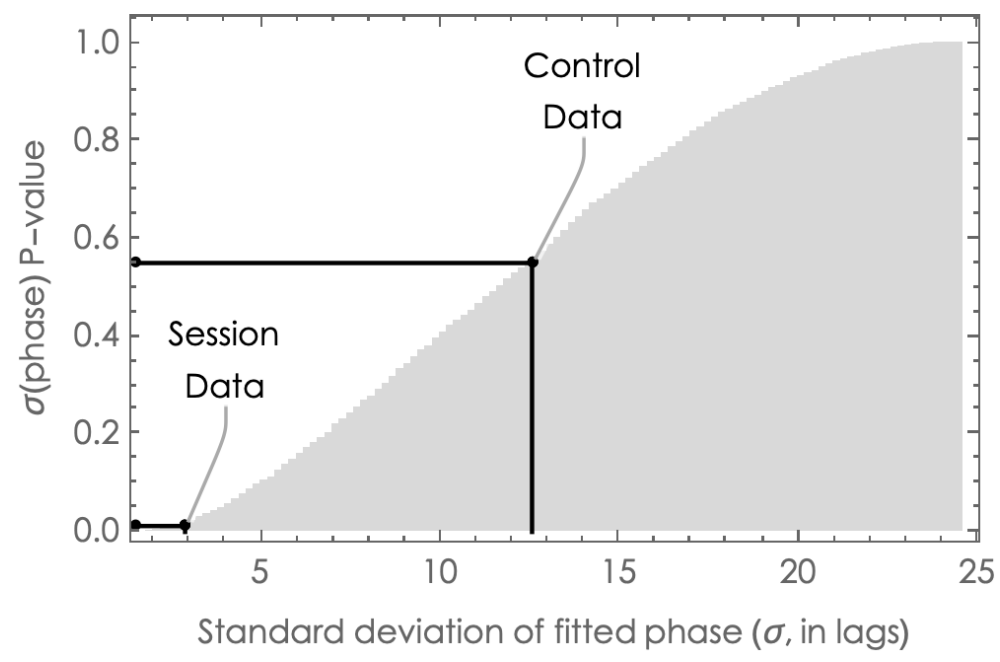

Figure A.3. The results and CDF for the test of H3. The horizontal axis is the bootstrapped standard deviation of the $\mathbf{\Delta S}$ lag curve phase. The vertical axis gives the cumulative probability, which is equivalent to the $p$-value for this test; $p$-value $=0.013$, one-tailed.

Determination of the $\sigma_{\phi}$ distribution increases calculation times by a factor of 100 , but yields in return a significant increase in the power to detect an effect. In particular, it is sensitive to effects that lock to the periodicity of the SCC, the alternating instructional conditions of concentrate and relax. In this case, the locking will narrow the variance of $\phi$, and one-sided tests for $\mathrm{H} 3$ predict a low value of $\sigma_{\phi}$ relative to the Null distribution. Figure A.4 shows the evolution of $\sigma_{\phi}$ as simulations include progressively stronger effects. 

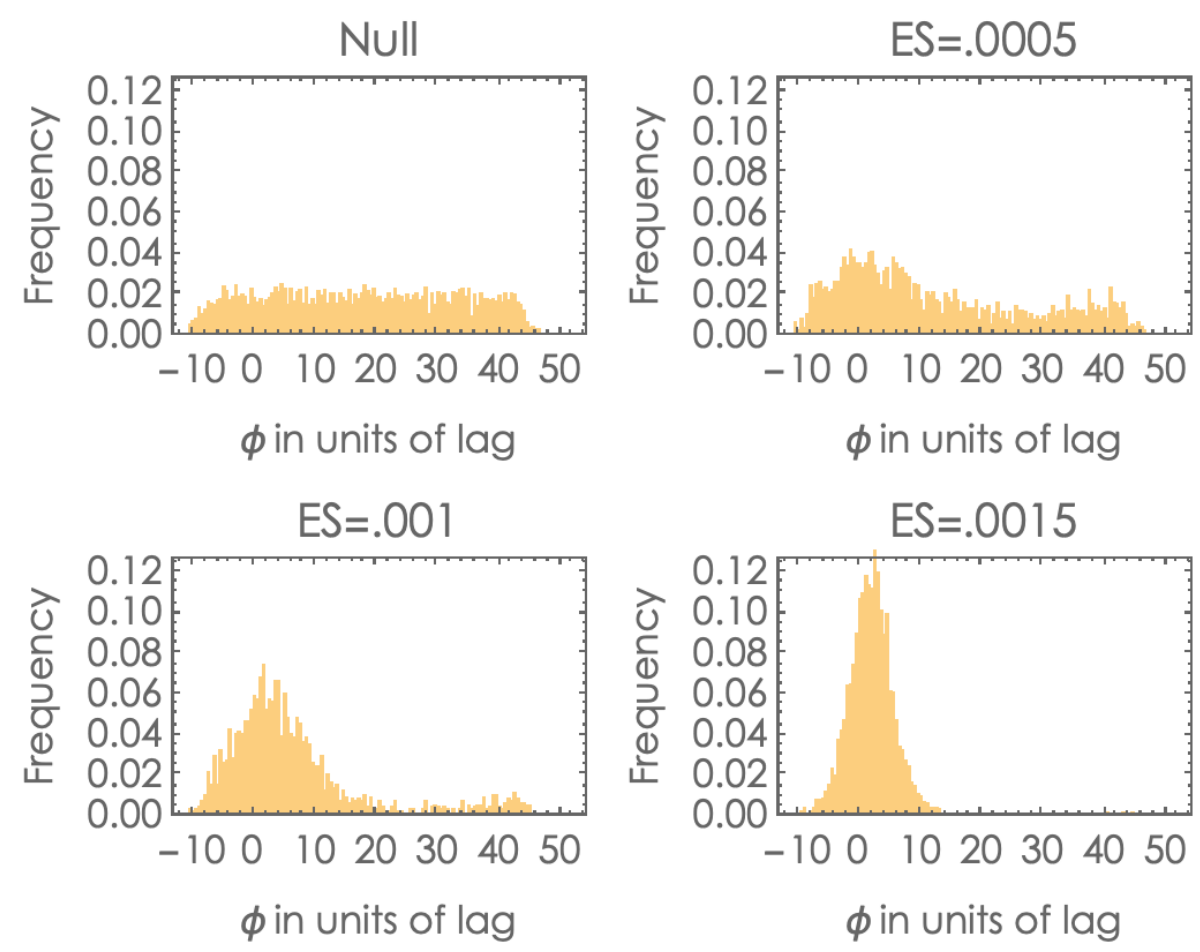

Figure A.4. Locking of the fit parameter $\phi$ under the presence of an effect as evidenced by the narrowing of its variability. The distribution of $\phi$ sharpens as a simulated psi effect with periodicity of the SCC is increased. The units of ES refer to relative differences of $S$-values under the concentrate and relax conditions. The IONS experiment yields an ES slightly smaller than 0.0015 .

\section{E. Methods for combining $p$-values}

The combined $p$-value for tests of $\mathrm{H} 1-\mathrm{H} 3$ are given by the $p$-value of Fisher's ChiSquare, $F c h i$. The value is determined by comparison of Fchi with its Null distribution, as estimated by Monte Carlo simulation. The procedure compensates for any dependencies between the triples of $p$-values associated with $\mathbf{m a x Z}, \mathbf{A}$ and $\sigma_{\phi}$. The statistics are, in fact, highly correlated (Spearman Ranks: $\max Z$ and $\mathbf{A} \approx 0.87$; $\max Z$ or $\mathbf{A}$ vs. $\sigma_{\phi} \approx-0.45$ ), so a Monte Carlo approach to Fchi is necessary. The Fchi value for the IONS session data is 29.9, which is higher than any value in the simulated distribution of 4000 Monte Carlo iterations. The control data yields an Fchi of 10.8. The corresponding P-value estimates are $<0.00021$ and 0.95 , respectively. A histogram of the Fchi Null distribution is shown in Figure 4 in the text.

The weighted Stouffer $\mathrm{Z}$ to combine tests from the independent IONS and IMI datasets is given by: 


$$
Z=\frac{\sum_{i=1}^{N} w_{i} Z_{i}}{\sqrt{\sum_{i=1}^{N} w_{i}^{2}}}
$$

where $\mathrm{Z}_{\mathrm{i}}$ are normal scores inverted from the test $p$-values and the relative weights, $\mathrm{w}_{\mathrm{i}}$, are 1 and 0.845 , respectively, for the IMI and IONS datasets. The weights are the square root of the ratio of total coincidence counts for the two datasets.

\section{F. Comments on the Monte Carlo and bootstrap procedures}

The analyses present estimated $p$-values of test statistics and these assume that simulations adequately represent the Null hypothesis. Given the complexity of the experiment, the data structure and the tests, some comments are in order to justify this assumption.

A modelling approach to surrogate Null data is likely to encounter mistaken assumptions or incorrect biases, so we adopt a bootstrap procedure using the experimental data to produce surrogate datasets by random sampling with replacement. There are several limitations on the sampling that must be considered. First, sampling on trials needs to respect the analyzer settings because indiscriminate sampling would erase the Bell correlations. Second, sessions have different average $\mathbf{S}$ levels, and this could conceivably produce accidental structure in analyses which should not be expunged from the surrogate datasets. Figure A.5, which shows independent $\mathbf{S}$-values from 7 different sessions, demonstrates that the mean levels of the $\mathbf{S}$-value vary considerably, as does the statistical noise in the measurements. However, fluctuations within a session are quite stable and generally lie within a 90\% CI of the noise.

Thus, we chose to constrain the random sampling to be from within a session and to respect polarization settings. Two ways to achieve this are 1) adding Poisson counting errors to each trial and, 2) randomly sampling (with replacement) the exact trials within each session and within each subset of analyzer settings. The two procedures generate distributions of test statistics for H1-H3 that are nearly identical (see Figure A.6). Distributions generated from experimental and control data are in similar close agreement. However, the Poission error method produces slightly less significant results at small $p$-values, and we have adopted this more conservative method in reporting our results. 


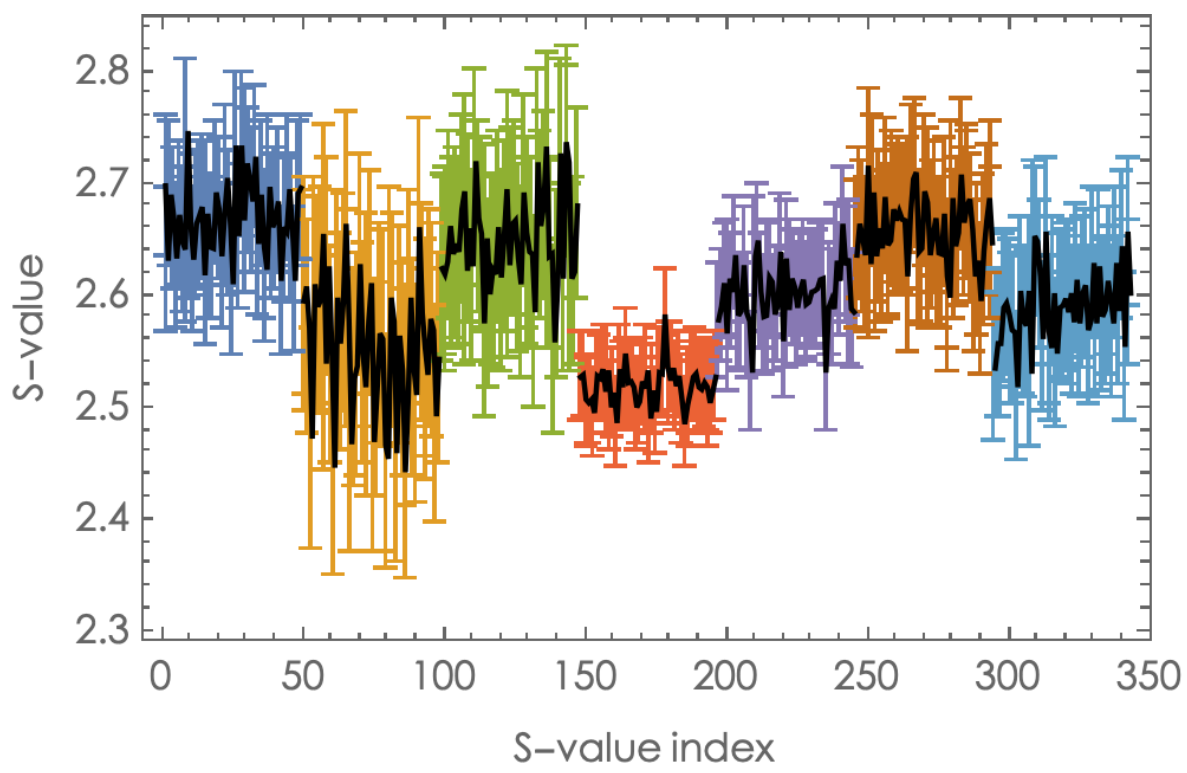

Figure A.5. Independent $\mathbf{S}$-samples taken at 16-trial intervals for 7 IONS experimental sessions. The error bars are $90 \%$ CIs for each sample. Differences in errors indicate inverse differences in the flux of coincidence counts, likely due to the laser power level or quality of the optical alignment.
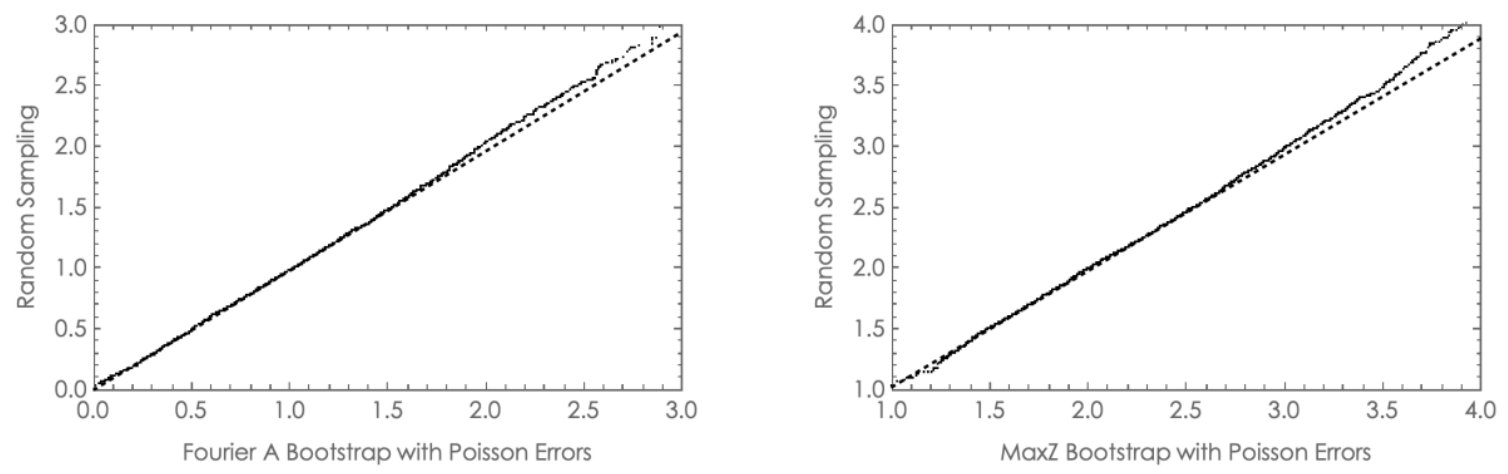

Figure A.6. Quantile-quantile plots of the distributions for maxZ and A comparing the two bootstrap methods. The random sampling method (vertical axis) is slightly more sensitive and produces more significant $p$-values at quantiles above $\approx 0.98$ (corresponding to $p$-values smaller than 0.02). 
For the Poisson error bootstrap, the average value of coincidence counts is tabulated for each setting of each session. The resulting $16 \mathrm{xN}$ matrix (where $\mathrm{N}$ is the number of sessions) is then used to generate a new Poisson random variable for each trial in the dataset, where the matrix element for the session and setting sets the trial's Poisson distribution parameter (i.e., the distribution mean).

A further step is needed to estimate the Null distribution of the fitted phase's variance. The procedure is essentially a bootstrap within a bootstrap, and is done as follows. First a surrogate dataset is generated using one of the methods above. The surrogate data are thus a concatenation of sessions, with the resamplings performed separately on each session. From the surrogate, the $\Delta \mathbf{S}$ lag curve is generated, and the test statistics $\max \mathbf{Z}, \mathbf{A}$ and $\phi$ are calculated. To estimate $\phi$ 's standard error, $\sigma_{\phi}$, a new dataset is generated by randomly sampling on the sessions (there are a total of 31 sessions in the three IONS experiments, for example). Statistics are calculated and the resampling on sessions is repeated (typically 100 to 200 times) to create a sample distribution of phases associated with each surrogate dataset. The phase standard error is then calculated and grouped with the values of the three test statistics for that surrogate dataset. The bootstrap thus generates four distributions for the four test statistics. The distributions are then used to transform the distribution values to $p$-values, and the $\operatorname{logs}$ of the grouped $p$-values for $\operatorname{maxZ}, \mathbf{A}$ and $\sigma_{\phi}$ are summed to give the distribution of $F$ chi. 